\title{
Étude ethnobotanique des plantes médicinales dans le Parc National de Talassemtane (Rif occidental du Maroc)
}

\author{
Mariam RHATTAS', Allal DOUIRA ${ }^{2}$ et Lahcen ZIDANE ${ }^{1}$ \\ ${ }^{1}$ Laboratoire de Biodiversité et Ressources Naturelle, Faculté des Sciences, Université Ibn Tofaïl, Kenitra, Maroc, \\ BP 133, \\ 2 Laboratoire de Botanique et de Protection des Plantes, Faculté des Sciences, Université Ibn Tofail, Kenitra, Maroc, \\ BP 133 \\ Auteur email correspondante : mariamrhattas@gmail.com
}

Original submitted in on $5^{\text {th }}$ November 2015. Published online at www.m.elewa.org on 31st January 2016

http://dx.doi.org/10.4314/jab.v97i1.5

\section{RÉSUMÉ :}

Objectif: Cette étude ethnobotanique a été menée dans le parc national de Talassemtane pour recueillir des informations sur les usages phyto thérapeutiques pratiqués dans cette région.

Méthodologie et résultats : A l'aide de 930 fiches questionnaires, une série d'enquêtes ethnobotaniques a été réalisée durant l'année 2014. L'identification des plantes récoltées sur le terrain a été effectuée au laboratoire. Le dépouillement des résultats a permis d'inventorier 103 espèces et 82 genres appartenant à 47 familles dont les plus représentées dans la région sont les Lamiaceae $(42,35 \%)$, Cistaceae $(30,60 \%)$, Asteraceae $(9,25 \%)$, et les Apocynaceae, Thymelaeaceae (8,90\%). Les espèces les plus utilisées par la population locale sont: Cistus varius et Marrubium vulgare, représentées respectivement par $14,59 \%$, et Mentha pulegium (12,46\%), Matricaria camomilla (9,25\%), ensuite viennent Nerium oleander, Daphne gnidium, et Cistus monspeliensis avec respectivement 8,90\%, après viennent Salvia officinalis $(7,83 \%)$, Lavandula dentata $(7,47 \%)$, et Cistus albidus $(7,12 \%)$. Les résultats montrent également que le feuillage constitue la partie la plus utilisée, avec un pourcentage de $71,75 \%$, et la majorité des remèdes sont préparé sous forme d'infusion et macération avec respectivement $30,43 \% ; 14,41 \%$. Par ailleurs, les maladies traitées par ces plantes médicinales sont dominées par les affections digestives $(37,78 \%)$, les affections cutanées $(15 \%)$, les affections génito-urinaires $(8,89 \%)$, et les affections neurologiques $(6,67 \%)$.

Conclusion et application de la recherche : Les enquêtes effectuées ont permis d'inventorier les espèces médicinales et de collecter le maximum d'information concernant les usages thérapeutiques traditionnels locaux. Ces résultats peuvent être considérés comme une source d'information pour les recherches scientifiques dans le domaine de la phyto-chimie et de la pharmacologie.

Mots-clés : Parc national de Talassemtane, Plantes médicinales, Ethnobotanique, Médecine traditionnelle. 

Morocco)

\section{ABSRACT}

Objective: This ethnobotanical study was conducted in the Talassemtane National Park to collect information on the phyto-therapeutic uses practiced in this region.

Methods and results: using questionnaires 930 sheets, a series of ethnobotanical surveys was conducted in the year 2014. The identification of plants collected in the field was conducted in the laboratory. The counting of the results allowed to inventory 103 species and 82 genera in 47 families, the most represented in the region are the Lamiaceae (42.35\%), Cistaceae (30.60\%), Asteraceae (9.25\%), and Apocynaceae, Thymelaeaceae are represented respectively by $(8.90 \%)$. The most used species by the local population are: Cistus and varius Marrubium vulgare represented by $14.59 \%$ respectively, and Mentha pulegium (12.46 \%), Matricaria camomilla (9.25\%), followed by Nerium oleander, Daphne gnidium and Cistus with monspeliensis $8.90 \%$ respectively, after coming Salvia officinalis (7.83\%), Lavandula dentata $(7.47 \%)$ and Cistus albidus (7.12\%). The results also show that the foliage is the most used part, with a percentage of $71.75 \%$, and the majority of remedies are prepared as an infusion and maceration with $30.43 \%$, respectively; $14.41 \%$. Moreover, diseases treated by these medicinal plants are dominated by digestive disorders $(37.78 \%)$, skin disorders $(15 \%)$, genitourinary diseases $(8.89 \%)$ and neurological disorders $(6$, $67 \%)$.

Conclusion and application of research: The investigations have made an inventory of medicinal plants and collect the maximum information regarding local traditional therapeutic uses. Ultimately, these results can be regarded as a source of information for scientific research in the field of phyto-chemistry and pharmacology.

Keywords : Natural Park Talassemtane, Medicinal plants, Ethnobotany, Traditional medicine.

\section{INTRODUCTION}

Depuis la nuit des temps, l'homme s'est employé à exploiter la nature pour ses besoins médicaux et alimentaires, et au cours du développement des anciennes civilisations l'exploitation des plantes à usage médicinale s'est développée grâce à leur savoir et à leur expérimentation effectués dans ce domaine. II a inventorié, déterminé et utilisé les plantes médicinales d'une façon plus ou moins correcte ; mais avec les changements qu'a connus l'humanité au niveau technologique et médicale, le souci d'utilisation des plantes médicinale et aromatique ne présentait plus un problème concernant les doses utilisées. Mais ceci n'est pas épargné des problèmes d'intoxication que peuvent présenter certaines plantes médicinales concernant l'utilisation non codifiée. Actuellement, cette médication par les plantes connaît un regain d'intérêt notable, grâce aux études scientifiques basées sur les méthodes analytiques et les expérimentations nouvelles, que le monde médical découvre de plus en plus, le bien-fondé des prescriptions empiriques des plantes médicinales (Lahsissene et al. 2009). Parmi les disciplines scientifiques qui s'intéressent à la phytothérapie traditionnelle, l'ethnobotanique est considérée comme une science qui permet de traduire le savoir-faire populaire en savoir scientifique. Les premières études sérieuses sur les plantes médicinales marocaines remontent au 1978 lorsqu'il a paru un livre de Jamal Bellakhdar intitulé : « Médecine traditionnelle et toxicologique ouest-saharienne ». Ce livre constitue une contribution incontestable à la pharmacopée marocaine. Bellakhdar a publié également en 1997 un travail de synthèse très importante intitulé : la pharmacopée marocaine traditionnelle. Benchaâbane \& Abbad (1997) et d'autres ont publié des travaux dans ce sens. Certains auteurs ont essayé de valoriser des huiles essentielles des plantes médicinales (Remmal, 1994 ; Bammi, 1995 ; Belkamel et al., 2008 et 2013 ; Tantaoui et al., 1993 a-b ; Bammi et al., 2000 ; Settaf et al., 

National de Talassemtane (Rif occidental du Maroc)

2000 ; Ouchbani, 2012 ; Bammou et al., 2015) et de déterminer leurs activités biologiques (Remmal, 1994 ; Bammi, 1995 ; Belkamel et al., 2008 et 2013). D'autres études se sont intéressées aux flores médicinales régionales dont les plus importantes ont été menées par Bellakhdar et al. (1987) dans la région de Tata (sud marocain), Benabid et Bellakhdar (1987) dans le Rif, Kahouadji (1995) dans le Maroc oriental, Bammi et

\section{MATÉRIEL ET MÉTHODES}

Diversité écologique, géologique et économique du Rif: Le Rif, édifice montagneux de la partie septentrionale du Maroc, fait partie des chaînes alpines du pourtour de la Méditerranée occidentale, par son âge et son style (Lespinasse, 1975). II comporte, audessus d'éléments du socle ancien, une série sédimentaire secondaire et tertiaire. Sa structure présente des plissements multiples et intenses accompagnés de métamorphisme ainsi que des déversements et des charriages importants orientés vers l'ouest et le Sud-ouest (Michard, 1976). Par rapport à l'ensemble des montagnes nord-africaines, la situation géographique privilégiée du Rif entre l'Atlantique et la Méditerranée lui confère une originalité climatique dont la combinaison avec la diversité orographique et géologique régionale favorise une grande richesse écologique (Benabid, 1983a). Dans la partie occidentale et centrale de ces montagnes, l'élévation attitudinale exposée à l'humidité et à la douceur des influences climatiques atlantiques et méditerranéennes favorise le développement d'une végétation forestière luxuriante. $\mathrm{Du}$ thermoméditerranéen à l'oroméditerranéen, l'étagement de cette végétation est très affecté par l'hétérogénéité des expositions et les substrats géologiques (Taiqui \& Martin, 1997). En plus de ces facteurs naturels, l'ancienneté de la présence humaine constitue une composante fondamentale dans la structuration et le fonctionnement de ces paysages. Le conservationnisme, caractéristique des montagnards, l'urbanisation historique qui remonte aux premières civilisations méditerranéennes, l'arabisation profonde et le rôle joué par la région dans le développement et l'épanouissement du soufisme marocain sont des constituants essentiels du paysage culturel rifain. $\grave{A}$ partir du XVI-XVlle siècles, suite à la stagnation économique du Maroc et son repli sur lui-même, la région a subi une régression générale vers un système économique de subsistance (Taiqui \& Martin, 1997).
Douira (2002) dans la région de Benslimane, Hseini et Kahoudji (2007) dans la région de Rabat, Mehdioui et Kahouadji (2007) dans la région d'Essaouira, Lahsissene et al. (2009) dans la région de Zaer, El Hassani et al. (2012) dans la Moyenne Moulouya. Le présent travail s'inscrit dans ce sens et concerne le recensement de la flore du Parc national de Talassemtane et la détermination des espèces à usage médicinale.

D'un système de demande de productions commerciales naturelles et agraires variées, le Rif est passé à une économie autarcique dont la relation avec l'extension de la végétation naturelle est démontrée par les analyses polliniques (Reille, 1977). Ces changements ont représenté au niveau du paysage la disparition de plusieurs centres urbains et l'organisation spatiale des agrosystèmes traditionnels sur de petites étendues limitées autour des hameaux et des petites villes persistantes. En considérant l'exemple du seul paysage autour de la ville de Chefchaouen, témoignage d'une histoire écologique riche et vivante, la haute diversité écologique, fortement concentrée dans l'espace, est représentée par une vallée majestueuse occupée par une subéraie étendue et dominée par les conifères et les forêts caducifoliées de montagne. Au niveau du massif calcaro-dolomitique de Talassemtane, Quezel et al. (1990), disent à propos du paysage endémique de la sapinière (Abies maroccana, Cedrus atlantica, Pinus nigra subsp. mauritanica, Acer granatensis, Quercus rotundifolia, Pinus pinaster subsp. maghrebiana), un véritable pôle de diversification des essences forestières en Afrique du Nord. Face à la dorsale calcaire, le massif gréseux de Bou-Hachem (Cedrus atlantica, Quercus pyrenaica, Quercus canariensis, Quercus rotundifolia, Pinus pinaster subsp. maghrebiana, Alnus glutinosa, Prunus Iusitanicum, tourbières à Sphagnum auriculatum...) représente une valeur biologique exceptionnelle et un intérêt biogéographique irremplaçable (Sauvage, 1958). En ce qui concerne les végétaux, parmi les grands écosystèmes forestiers du Rif, les oléastraies (Olea europaea var. silvestris) qui occupaient les sols fertiles à basses altitudes sont totalement éteintes et ne sont épargnées qu'au niveau des vestiges maraboutiques (Benabid, 1987). Les cocciféraies, qui formaient de véritables forêts au Rif occidental, ne sont plus visibles qu'au niveau des marabouts ou sousforme de matorrals (Benabid, 1984). La situation des 


\section{Rhattas et al. J. Appl. Biosci. 2016 Étude ethnobotanique des plantes médicinales dans le Parc National de Talassemtane (Rif occidental du Maroc)}

tétraclinaies et des pinèdes naturelles n'est pas très différente. Quant aux subéraies et chênaies vertes rifaines restent assez étendues, mais fortement dégradées par différents modes d'exploitation et de dégradation. $A$ haute altitude, la sapinière risque de disparaître dans un avenir très proche (Benabid, 1987 et 1991). Le Parc national de Talassemtane s'étend sur une superficie de 58.950 ha est majoritairement incluse dans la province de Chefchaouen et une petite partie se situe sur la province de Tetouan (Tableau 1). Sur les 34 communes que compte la province de Chefchaouen, 6 sont concernées par l'installation du parc. Elles dépendent de deux cercles, Bab Taza et Bou Ahmed, et 4 caïdats, Bab Taza, Talembote, Assifane et Bou Ahmed (PDAGPNT, 2004).

Tableau 1 : Découpage administratif du Parc national de Talassemtane

\begin{tabular}{|c|c|c|c|}
\hline Province & Cercle & Caïdat & Commune \\
\hline \multirow{6}{*}{ Chefchaouen } & \multirow{2}{*}{ Bab Taza } & \multirow{2}{*}{ Bab Taza } & Bab Taza \\
\hline & & & Beni Derkoul \\
\hline & \multirow{4}{*}{ Bou Ahmed } & \multirow{2}{*}{ Talembote } & Talembote \\
\hline & & & Tassift \\
\hline & & Assifane & Beni Selmane \\
\hline & & Bou Ahmed & Steha \\
\hline \multirow{4}{*}{ Tétouan } & \multirow{4}{*}{ Tétouan } & Al Ouad & Al Ouad \\
\hline & & Al Hamra & Al Hamra \\
\hline & & Bni Said & Bni Said \\
\hline & & Oulad Ali Mansour & Oulad Ali Mansour \\
\hline
\end{tabular}

Méthodologie: L'étude ethnobotanique des plantes médicinales du Parc national de Talassemtane a été effectuée d'une part selon un plan d'échantillonnage stratifié et d'autre part à l'aide d'une fiche questionnaire répartie sur la totalité du Parc pour avoir une vue d'ensemble sur les utilisations traditionnelles locales et sur la diversité floristique de la pharmacopée de cette zone. Grâce aux 930 fiches questionnaires (Annexe 1), qui ont été effectuées au niveau de 31 villages avec $\mathrm{N}$ $=30$ personnes/village, réparties dans le Parc national de Talassemtane (tableau $n^{\circ} 2$ ), afin de couvrir la totalité de la surface étudiée en récoltant le maximum d'information sur l'utilisation des plantes médicinales.
Ce travail a été réalisé entre le mois de Mars et le mois d'Août (2014) durant cinq missions de terrain (Avril, Mai, Juin, Juillet, Août) de 5 jours consécutifs. Cette enquête a été réalisée chez la population locale pour définir les plantes à usage médicinales selon le sexe, le niveau académique des enquêtés, la situation familiale, la profession, le mode d'utilisation des plantes, la partie utilisée et les maladies traitées par ces plantes. L'enquête a permis d'interroger 930 personnes âgées de 20 à plus que 70 ans, répartis en 532 femmes contre 398 hommes et à des niveaux intellectuels différents, qui nous ont informés sur les applications thérapeutiques et traditionnelles locales dans le Parc.

Tableau 2 : Répartition des enquêtes ethnobotaniques selon les zones, effectuées début de Mars et fin Août 2014 ;

\begin{tabular}{llll}
\hline Province & Zone d'enquête & Nom de zone & Nombre d'enquêtes \\
\hline & Parc national de Talassemtane & & \\
\hline Chefchaouen & Zone 1 & Bab Taza & 30 \\
\hline Zone 2 & Zaouia & 30 \\
\hline Zone 3 & Tissouka & 30 \\
\hline Zone 4 & Mechkrala & 30 \\
\hline Zone 5 & Azilan & 30 \\
\hline Zone 6 & Afaska & 30 \\
\hline Zone 7 & Imezar & 30 \\
\hline
\end{tabular}




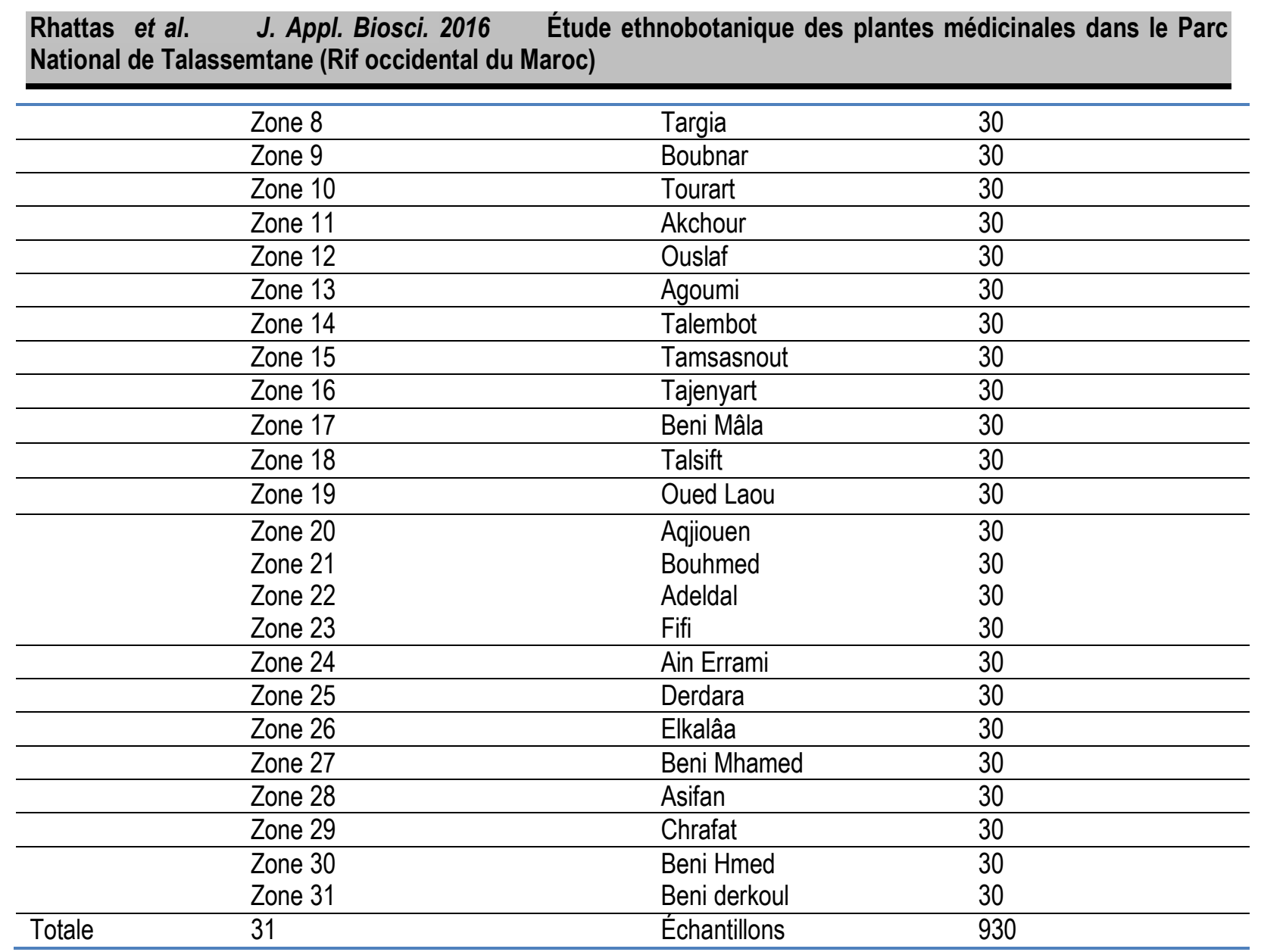

\section{RÉSULTATS ET DISCUSSION}

Selon les enquêtes réalisées, nous avons rapporté 184 plantes récoltées au Parc. Ces plantes sont réparties en 47 familles, 103 espèces végétales et 82 genres. Concernant leur utilisation comme plantes médicinales, les résultats obtenus ont montré que ce sont les femmes qui les utilisent plus, ce résultat est conforme aux ceux signalés par Kahouadji (1995) et Hmamouchi (2001).

Profil des enquêtés: Le traitement statistique des données a permis de noter (Figures 1, 2, 3, 4 et 5), à l'échelle des enquêtés du Parc national de Talassemtane, que parmi les $57 \%$ des femmes et $43 \%$ des hommes recensés, une grande catégorie des jeunes personnes de classe d'âge [20 à 39 ans] ont une fréquence d'utilisation des plantes médicinales de $47 \%$, viennent ensuite les tranches d'âge [40 à 59 ans], [60 à 79 ans], avec respectivement $36 \%$ et $17 \%$, ceci est dû au redressement de personnes âgées dans le monde rural, ce qui influence les résultats obtenus. En fait, la majorité des personnes enquêtées sont mariées avec $55,56 \%$, soit $54,08 \%$ sont des paysans et $56,12 \%$ sont des analphabètes en relation directe avec la nature (pâturage et agriculture), ce qui explique la catégorie des jeunes personnes et surtout les femmes qui ont acquis une bonne connaissance pour l'utilisation des plantes médicinales. Sur le terrain d'enquête, les femmes et les hommes se chargent équitablement de la collecte des plantes médicinales, le séchage, et le stockage, mais la collecte dans les zones réputées dangereuses, est effectuée seulement par les hommes (Mehdioui \& Kahouadji, 2007). La préparation des recettes pour les soins des membres de la famille est effectuée par les femmes. 
Rhattas et al. J. Appl. Biosci. 2016 Étude ethnobotanique des plantes médicinales dans le Parc National de Talassemtane (Rif occidental du Maroc)

Répartition des enquêtés selon l'âge

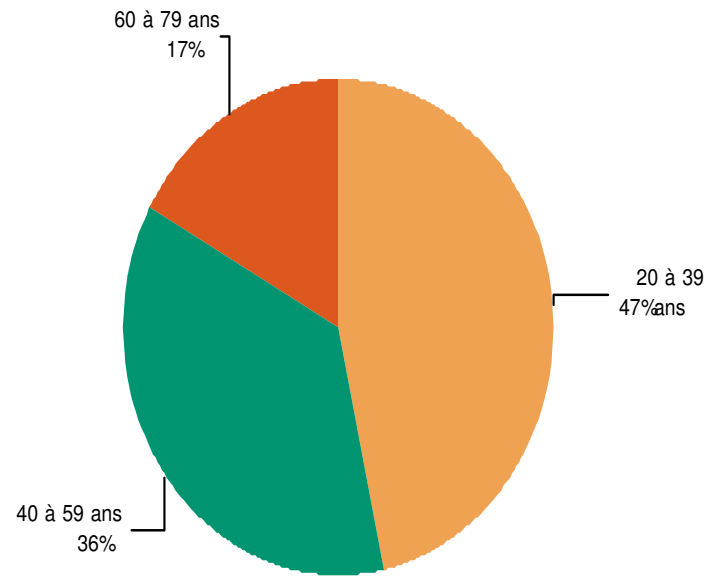

Figure 1 : Classe d'âge selon le nombre des personnes enquêtés (Enquête début mois de mars au fin août 2014)

\section{Répartition des enquêtés selon le sexe}

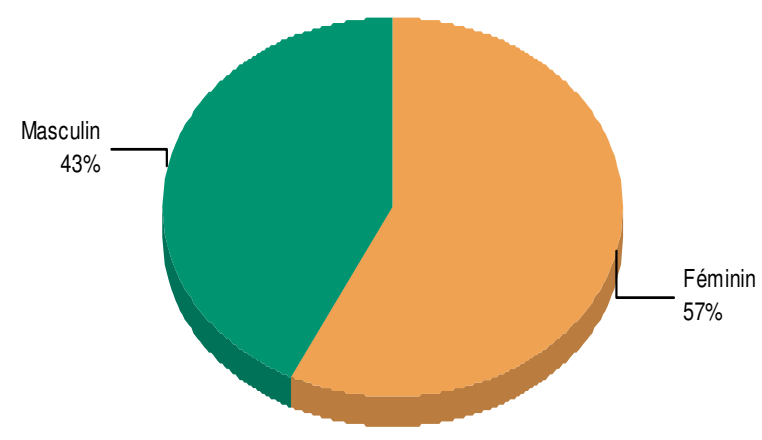

Figure 2 : Répartition des enquêtés selon le sexe (Enquête début mois de mars au fin août 2014) 
Rhattas et al. J. Appl. Biosci. 2016 Étude ethnobotanique des plantes médicinales dans le Parc National de Talassemtane (Rif occidental du Maroc)

Répartition des enquêtés selon la situation familiale

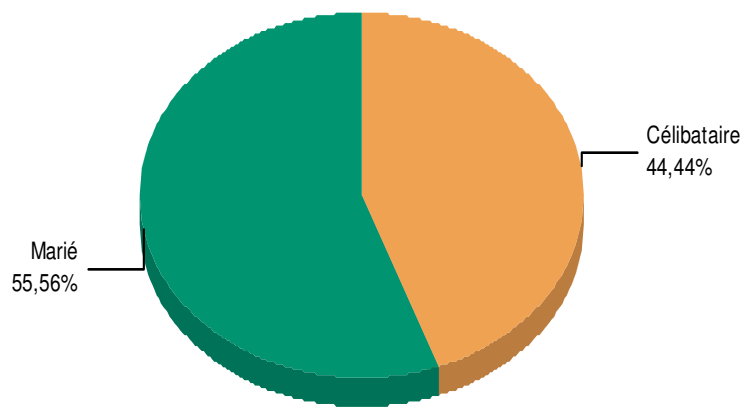

\begin{tabular}{|rr}
\hline Célibataire & $44,44 \%$ \\
\hline Marié & $55,56 \%$ \\
Total : & $100,00 \%$ \\
\hline
\end{tabular}

Figure 3 : Situation familiale selon les enquêtés (Enquête début mois de mars au fin août 2014);

\section{Répartition des enquêtés selon la profession}
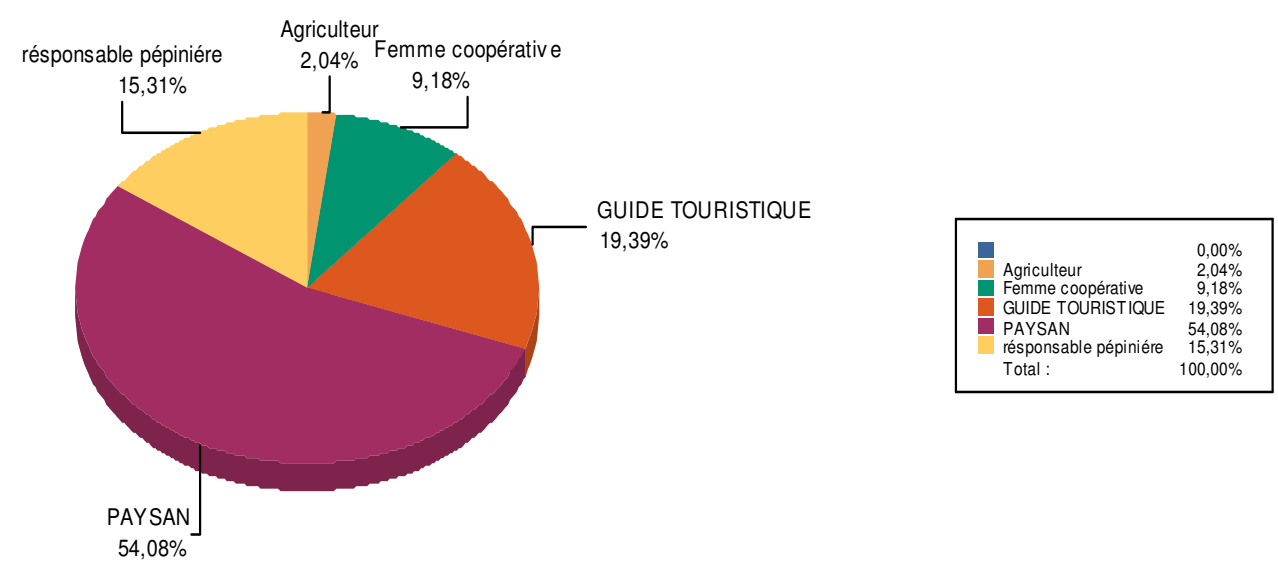

Figure 4 : Profession des enquêtés (Enquête début mois de mars au fin août 2014); 
Rhattas et al. J. Appl. Biosci. 2016 Étude ethnobotanique des plantes médicinales dans le Parc National de Talassemtane (Rif occidental du Maroc)

\section{Répartition des enquêtés selon le niveau d'études}

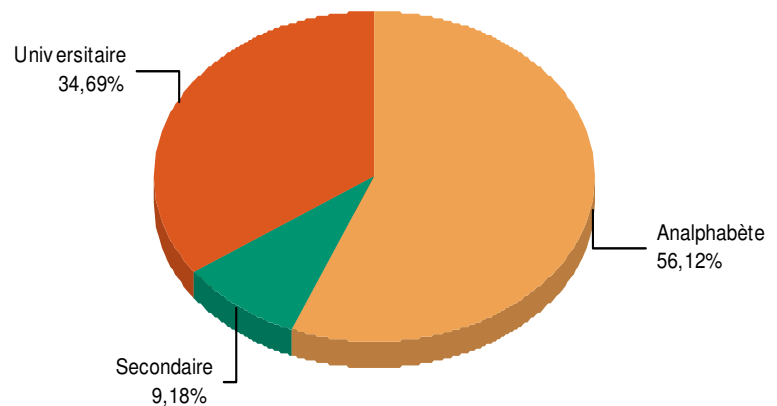

Figure 5 : Niveau d'étude des enquêtés (Enquête début mois de mars au fin août 2014) ; 


\section{Rhattas et al. J. Appl. Biosci. 2016 Étude ethnobotanique des plantes médicinales dans le Parc National de Talassemtane (Rif occidental du Maroc)}

Tableau 3 : Liste des plantes médicinales recensées dans le Parc national de Talassemtane

\begin{tabular}{|c|c|c|c|c|c|c|c|}
\hline $\mathrm{N}^{\circ}$ & Familles & Espèces & Nom Français & $\begin{array}{l}\text { Nom } \\
\text { Vernaculaire }\end{array}$ & $\begin{array}{l}\text { Usage de la } \\
\text { plante }\end{array}$ & $\begin{array}{l}\text { Mode de } \\
\text { préparation }\end{array}$ & Type de maladie \\
\hline 1 & Acanthaceae & Acanthus mollis & $\begin{array}{l}\text { Acanthe à feuille } \\
\text { molle }\end{array}$ & El akneth & Thérapeutique & Infusion/Cataplasme & Soulager les brûlures \\
\hline 2 & Adiantaceae & $\begin{array}{l}\text { Adiantum } \\
\text { capillus-veneris }\end{array}$ & $\begin{array}{l}\text { Capillaire de } \\
\text { Montpellier }\end{array}$ & $\begin{array}{l}\text { Kouzborat El } \\
\text { bir }\end{array}$ & Thérapeutique & Sirop & $\begin{array}{l}\text { Utilisées en phytothérapie pour les } \\
\text { problèmes de voies respiratoires. }\end{array}$ \\
\hline 3 & Anacardiaceae & Pistacia lentiscus & $\begin{array}{l}\text { Pistachier } \\
\text { lentisque }\end{array}$ & Drou - Afadiz & $\begin{array}{l}\text { Thérapeutique/ } \\
\text { Cosmétique }\end{array}$ & Décoction & $\begin{array}{l}\text { Affection oculaire Soin des cheveux } \\
\text { Affection cutané Antimigraineux }\end{array}$ \\
\hline 4 & \multirow{3}{*}{ Apiaceae } & Ammi majus & $\begin{array}{l}\text { Ammi commun/ la } \\
\text { fleur de l'évêque }\end{array}$ & Belala & Thérapeutique & Infusion/ Décoction & $\begin{array}{l}\text { Anti-dermatosique, diurétique, utilisée } \\
\text { pour le traitement du vitiligo }\end{array}$ \\
\hline 5 & & $\begin{array}{l}\text { Eryngium } \\
\text { bourgatii }\end{array}$ & Panicaut & $\begin{array}{l}\text { Ezourayka - } \\
\text { chouka zarka }\end{array}$ & Thérapeutique & Cuit & Affection digestive \\
\hline 6 & & $\begin{array}{l}\text { Eryngium } \\
\text { triquetrum }\end{array}$ & & & Thérapeutique & Décoction & $\begin{array}{l}\text { Affection Intestinale, Refroidissement, } \\
\text { amygdalite }\end{array}$ \\
\hline 7 & Apocynaceae & Nerium oleander & Laurier-rose & Dafla & Thérapeutique & Cataplasme & $\begin{array}{l}\text { Elle est reconnue par sa toxicité, et les } \\
\text { quelques expériences menées dans la } \\
\text { phytothérapie Les substances } \\
\text { dérivées de lauriers roses ont été } \\
\text { étudiés comme un traitement pour le } \\
\text { cancer, sans succès. [1] mais selon } \\
\text { notre enquête les habitants ont } \\
\text { déclaré que cette plante est utilisée } \\
\text { contre la gale, soin des cheveux, } \\
\text { coliques, brûlure, rhumes }{ }^{1}\end{array}$ \\
\hline
\end{tabular}

1 [1] Henary, HA; Kurzrock, R; Falchook, GS; et al. (2011). "Résultats finaux d'un First-in-humain étude de phase 1 du PBI-05204, et un inhibiteur de l'AKT, FGF-2, NF-Kb et P70S6K dans avancées patients atteints de cancer". J Clin Oncol 29 (supplément; abstraite 3023).

Newman, RA; Yang, P .; Pawlus, AD; Bloquer, KI (2008). "Glycosides cardiaques en tant que nouveaux agents thérapeutiques du cancer". Les interventions moléculaires 8 (1): 36-49 doi: 10,1124 / mi.8.1.8. PMID 18332483. 
Rhattas et al. J. Appl. Biosci. 2016 Étude ethnobotanique des plantes médicinales dans le Parc National de Talassemtane (Rif occidental du Maroc)

\begin{tabular}{|c|c|c|c|c|c|c|c|}
\hline $\mathbf{N}^{\circ}$ & Familles & Espèces & Nom Français & $\begin{array}{l}\text { Nom } \\
\text { Vernaculaire }\end{array}$ & $\begin{array}{l}\text { Usage de la } \\
\text { plante }\end{array}$ & $\begin{array}{l}\text { Mode de } \\
\text { préparation }\end{array}$ & Type de maladie \\
\hline 8 & Aristolochiaceae & $\begin{array}{l}\text { Aristolochia longa } \\
\text { (ou Baetica) }\end{array}$ & $\begin{array}{l}\text { Aristoloche } \\
\text { d'Afrique }\end{array}$ & Berztem & Thérapeutique & Cataplasme & $\begin{array}{l}\text { Affection Cutané, des Affections } \\
\text { intestinales, }\end{array}$ \\
\hline 9 & Asparagaceae & Ruscus aculeatus & Petit Houx & El ass Bari & $\begin{array}{l}\text { Thérapeutique/ } \\
\text { Cosmétique }\end{array}$ & Cuit/ Macération & $\begin{array}{l}\text { Contre la jaunisse, la chlorose et les } \\
\text { varices diurétiques }\end{array}$ \\
\hline 10 & Aspleniaceae & $\begin{array}{l}\text { Asplenium } \\
\text { adiantum-nigrum }\end{array}$ & Doradille noire & Sourkhous & Thérapeutique & Infusion & Affection Rénale \\
\hline 11 & & $\begin{array}{l}\text { Achillea } \\
\text { millefolium }\end{array}$ & $\begin{array}{l}\text { Verveine de } \\
\text { rocher }\end{array}$ & Louiza lehjar & Thérapeutique & Infusion & $\begin{array}{l}\text { Affection neurologique et les douleurs } \\
\text { intestinales }\end{array}$ \\
\hline 12 & & $\begin{array}{l}\text { Artemisia herba } \\
\text { alba }\end{array}$ & armoise blanche & Chih & Thérapeutique & Infusion & Colique intestinale, diarrhée, migraine \\
\hline 13 & Asteraceae & $\begin{array}{l}\text { Calendula } \\
\text { officinalis }\end{array}$ & $\begin{array}{l}\text { Soussi ou } \\
\text { calendule }\end{array}$ & $\begin{array}{l}\text { Zahra } \\
\text { Okehouan }\end{array}$ & $\begin{array}{l}\text { Thérapeutique/ } \\
\text { Cosmétique }\end{array}$ & Cataplasme & $\begin{array}{l}\text { Anti-inflammatoire, hypotenseur, } \\
\text { spasmolytique, immunostimulant et } \\
\text { hypo-cholestérolémiant. En usage } \\
\text { externe (teinture mère), c'est un } \\
\text { excellent anti-inflammatoire (peau et } \\
\text { muqueuses, intimes ou non), anti- } \\
\text { œdémateux et antivenimeux. II est } \\
\text { antibactérien, antiviral, antifongique et } \\
\text { cicatrisant² }^{2}\end{array}$ \\
\hline 14 & & $\begin{array}{l}\text { Carduus } \\
\text { Martinezii }\end{array}$ & Cardon & $\begin{array}{l}\text { El kardous - } \\
\text { Echouk }\end{array}$ & Thérapeutique & Décoction & Affection cutanée et abcès \\
\hline 15 & & Carduus nutans & Chardon penché & $\begin{array}{l}\text { Quouque El } \\
\text { bari }\end{array}$ & Thérapeutique & Huile essentielle & Purifie le sang \\
\hline 16 & & $\begin{array}{l}\text { Centaureae } \\
\text { clementei }\end{array}$ & Centaurée & $\begin{array}{l}\text { El } \\
\text { kantouryoune }\end{array}$ & Thérapeutique & Décoction & Fièvre \\
\hline 17 & & Cynara baetica & Cardon & $\begin{array}{l}\text { Quouque baldi } \\
\text { - El quanarya } \\
\text { - Kharchouf }\end{array}$ & Thérapeutique & Cuit & Affection digestive \\
\hline
\end{tabular}

2 Maria Treben, La Santé à la Pharmacie du Bon Dieu - conseils et pratique des simples (des plantes médicinales) [archive]. Éditeur W. Ennsthaler, Autriche, 112 p., (ISBN 3850681238). Première édition : 1983. Mauve : pp. 31-33. 

Rhattas et al. J. Appl. Biosci. 2016 Étude ethnobotanique des plantes médicinales dans le Parc National de Talassemtane (Rif occidental du
Maroc)

\begin{tabular}{|c|c|c|c|c|c|c|c|}
\hline $\mathbf{N}^{\circ}$ & Familles & Espèces & Nom Français & $\begin{array}{l}\text { Nom } \\
\text { Vernaculaire }\end{array}$ & $\begin{array}{l}\text { Usage de la } \\
\text { plante }\end{array}$ & $\begin{array}{l}\text { Mode de } \\
\text { préparation }\end{array}$ & Type de maladie \\
\hline 18 & Asteraceae & $\begin{array}{l}\text { Eupatorium } \\
\text { cannabinum }\end{array}$ & $\begin{array}{l}\text { Eupatoire } \\
\text { chanvrine }\end{array}$ & El Kahra & Thérapeutique & Macération & $\begin{array}{l}\text { Traitement des troubles du foie ou des } \\
\text { reins }{ }^{3} \text { mais selon les enquêtés ont } \\
\text { déclaré qu'ils l'utilisent dans le } \\
\text { traitement. }\end{array}$ \\
\hline 19 & & $\begin{array}{l}\text { Matricaria } \\
\text { chamomilla }\end{array}$ & Camomille & $\begin{array}{l}\text { Babounej- } \\
\text { Amlal }\end{array}$ & $\begin{array}{l}\text { Thérapeutique/ } \\
\text { Cosmétique }\end{array}$ & $\begin{array}{l}\text { Infusion/ } \\
\text { Macération }\end{array}$ & $\begin{array}{l}\text { Affection oculaire Soin des cheveux, } \\
\text { Affection neurologique }\end{array}$ \\
\hline 20 & Asparagaceae & Drimia maritima & Scille maritime & $\begin{array}{l}\text { Bsel Dib } \\
=\text { Elânsal }\end{array}$ & Cosmétique & Macération & $\begin{array}{l}\text { Utilisée à de faible dose pour les soins } \\
\text { des cheveux }\end{array}$ \\
\hline 21 & Blechnaceae & Blechnum spicant & & Sourkhous & Thérapeutique & Infusion & Affection Rénale \\
\hline 22 & Roraninareas & $\begin{array}{l}\text { Anchusa Azurea } \\
\text { ou Italica }\end{array}$ & $\begin{array}{l}\text { Buglosse bleue } \\
\text { ou d'Italie }\end{array}$ & & $\begin{array}{l}\text { Thérapeutique/ } \\
\text { Cosmétique }\end{array}$ & $\begin{array}{l}\text { Décoction/ } \\
\text { Cataplasme }\end{array}$ & $\begin{array}{l}\text { Affection rénale, hémorragies nasales, } \\
\text { fièvre, Comestibles, cicatrisante }\end{array}$ \\
\hline 23 & Boragınaceae & Borago officinalis & $\begin{array}{l}\text { Bourrache } \\
\text { officinale }\end{array}$ & $\begin{array}{l}\text { El hricha - } \\
\text { Lsan toure }\end{array}$ & Thérapeutique & Cuit & Affection digestive \\
\hline 24 & Brassicaceae & $\begin{array}{l}\text { Alyssum } \\
\text { spinosum }\end{array}$ & $\begin{array}{l}\text { Passerage } \\
\text { épineux }\end{array}$ & $\begin{array}{l}\text { Alousen - } \\
\text { Hachichat } \\
\text { soulahfat }\end{array}$ & Thérapeutique & Décoction & Fièvre, maux de tête \\
\hline 25 & Buxaceae & Buxus balearica & $\begin{array}{l}\text { Buis des } \\
\text { Baléares }\end{array}$ & El baks & pâturage & Cru & Alimentation pour bétail \\
\hline 26 & Cactaceae & Opuntia maxima & $\begin{array}{l}\text { Figuier d'Inde ; } \\
\text { Cactus }\end{array}$ & $\begin{array}{l}\text { Handia - } \\
\text { Karmous } \\
\text { Nsara }\end{array}$ & $\begin{array}{l}\text { Thérapeutique/ } \\
\text { Cosmétique }\end{array}$ & Cru & Affection digestive, soin des cheveux \\
\hline 27 & Cannabaceae & Cannabis sativa & Chanvre cultivé & Kif & $\begin{array}{l}\text { Thérapeutique/ } \\
\text { Cosmétique }\end{array}$ & $\begin{array}{l}\text { Cataplasme/ } \\
\text { Fumigation }\end{array}$ & $\begin{array}{l}\text { Soin des cheveux, calmante, sédative, } \\
\text { drogue }\end{array}$ \\
\hline 28 & Caryophyllaceae & Silene vulgaris & Silene enflé & Tirecht & $\begin{array}{l}\text { Thérapeutique/ } \\
\text { Lavage de laine }\end{array}$ & Décoction & $\begin{array}{l}\text { Contient du Saponine et il est utilisé } \\
\text { comme adjuvant, régulier certains } \\
\text { cycles hormonaux }\end{array}$ \\
\hline
\end{tabular}

${ }^{3}$ L'équipe des enseignants du DUMENAT Phytothérapie (faculté de médecine Paris-XIII, Bobigny), Phytothérapie, la santé par les plantes, Sélection du Reader's Digest, coll. Vidal, (ISBN 2709818515) 
Rhattas et al. J. Appl. Biosci. 2016 Étude ethnobotanique des plantes médicinales dans le Parc National de Talassemtane (Rif occidental du Maroc)

\begin{tabular}{|c|c|c|c|c|c|c|c|}
\hline $\mathbf{N}^{\circ}$ & Familles & Espèces & $\begin{array}{l}\text { Nom } \\
\text { Français }\end{array}$ & $\begin{array}{l}\text { Nom } \\
\text { Vernaculaire }\end{array}$ & $\begin{array}{l}\text { Usage de la } \\
\text { plante }\end{array}$ & $\begin{array}{l}\text { Mode de } \\
\text { préparation }\end{array}$ & Type de maladie \\
\hline 29 & \multirow{8}{*}{ Cistaceae } & Cistus albidus & $\begin{array}{l}\text { Ciste } \\
\text { blanchâtre }\end{array}$ & $\begin{array}{l}\text { Maknan - El } \\
\text { querida - } \\
\text { Markil-Kastous }\end{array}$ & $\begin{array}{l}\text { Thérapeutique/ } \\
\text { Cosmétique }\end{array}$ & Cru & Affection cutanée/ Affection digestive \\
\hline 30 & & Cistus crispus & $\begin{array}{l}\text { Ciste à f. } \\
\text { crispées }\end{array}$ & $\begin{array}{l}\text { Tarkal - } \\
\text { Shtapa - Lkrida }\end{array}$ & Thérapeutique & $\begin{array}{l}\text { Macération/ } \\
\text { Cataplasme }\end{array}$ & Affection cutanée/ Affection digestive \\
\hline 31 & & Cistus ladanifer & $\begin{array}{l}\text { Ciste porte- } \\
\text { ladanum }\end{array}$ & $\begin{array}{l}\text { Tarkal - } \\
\text { Shtapa - Lkrida }\end{array}$ & Thérapeutique & Distillation & Utilisée en parfumerie. \\
\hline 32 & & Cistus libanotis & $\begin{array}{l}\text { Ciste du } \\
\text { Liban }\end{array}$ & $\begin{array}{l}\text { Tarkal - } \\
\text { Shtapa - Lkrida }\end{array}$ & Thérapeutique & Macération & Affection cutanée \\
\hline 33 & & $\begin{array}{l}\text { Cistus } \\
\text { monspeliensis }\end{array}$ & $\begin{array}{l}\text { Ciste de } \\
\text { Montpelier }\end{array}$ & $\begin{array}{l}\text { Meril-Stevia- } \\
\text { Markil-Amzil - } \\
\text { shtapa }\end{array}$ & $\begin{array}{l}\text { Pesticide/ } \\
\text { Thérapeutique }\end{array}$ & $\begin{array}{l}\text { Cataplasme/ } \\
\text { Infusion }\end{array}$ & $\begin{array}{l}\text { Lutte contre les insectes/ Maladie de } \\
\text { diabète et pour faire le régime } \\
\text { Affectation digestive }\end{array}$ \\
\hline 34 & & Cistus salviifolius & $\begin{array}{l}\text { Ciste à feuille } \\
\text { de sauge }\end{array}$ & $\begin{array}{l}\text { El korida - } \\
\text { Stapa blanc }\end{array}$ & Thérapeutique & Infusion & Cultivé comme plante ornementale \\
\hline 35 & & $\begin{array}{l}\text { Cistus varius } \\
\text { Pourret }\end{array}$ & Ciste & $\begin{array}{l}\text { Tarkal - } \\
\text { Shtapa - } \\
\text { Lkrida }\end{array}$ & Thérapeutique & $\begin{array}{l}\text { Macération/ } \\
\text { Cataplasme }\end{array}$ & Non déterminé \\
\hline 36 & & Halimium calycinum & & & Non déterminé & Non déterminé & Non déterminé \\
\hline 37 & Cleomaceae & Cleome violacea & $\begin{array}{l}\text { Cleome } \\
\text { d'arabie }\end{array}$ & Mkhinza & Thérapeutique & Infusion & Contre la fièvre \\
\hline 38 & Convolvulaceae & $\begin{array}{l}\text { Calystegia silvatica } \\
\text { (kit.) griseb. }\end{array}$ & $\begin{array}{l}\text { Liseron des } \\
\text { haies }\end{array}$ & $\begin{array}{l}\text { Louaya - } \\
\text { Liblab }\end{array}$ & Non déterminé & Non déterminé & Non déterminé \\
\hline
\end{tabular}

\begin{tabular}{|l|l|l|l|l|l|l|l|}
\hline $\mathbf{N}^{\circ}$ & Familles & Espèces & $\begin{array}{l}\text { Nom } \\
\text { Français }\end{array}$ & $\begin{array}{l}\text { Nom } \\
\text { Vernaculaire }\end{array}$ & Usage de la plante & $\begin{array}{l}\text { Mode de } \\
\text { préparation }\end{array}$ & Type de maladie \\
\hline 39 & Convolvulaceae & $\begin{array}{l}\text { Convolvulus } \\
\text { althaeoides }\end{array}$ & $\begin{array}{l}\text { Liseron des } \\
\text { champs }\end{array}$ & Elouaya & Thérapeutique & Décoction/Infusion & Utilisé comme pesticide \\
\hline 40 & \multirow{2}{*}{ Crassulaceae } & Sedum acre & $\begin{array}{l}\text { Orpin âcre, } \\
\text { Joubarbe }\end{array}$ & $\begin{array}{l}\text { Zenbou - Hay } \\
\text { Elâlam }\end{array}$ & Non déterminé & Non déterminé & Non déterminé \\
\cline { 3 - 6 } 41 & & Sedum & Joubarbe & Zenbou - Hay & Non déterminé & Non déterminé & Non déterminé \\
\hline
\end{tabular}


Rhattas et al. J. Appl. Biosci. 2016 Étude ethnobotanique des plantes médicinales dans le Parc National de Talassemtane (Rif occidental du Maroc)

\begin{tabular}{|c|c|c|c|c|c|c|c|}
\hline & & forsterianum & & Elâlam & & & \\
\hline 42 & & $\begin{array}{l}\text { Sedum } \\
\text { hirsutum }\end{array}$ & & & Non déterminé & Non déterminé & Non déterminé \\
\hline 43 & \multirow{3}{*}{ Cupressaceae } & $\begin{array}{l}\text { Juniperus } \\
\text { communis }\end{array}$ & $\begin{array}{l}\text { Genévrier } \\
\text { commun }\end{array}$ & Elarâr - Amzi & Thérapeutique & Infusion & $\begin{array}{l}\text { À des effets diurétiques, } \\
\text { stomachiques et digestifs }\end{array}$ \\
\hline 44 & & $\begin{array}{l}\text { Juniperus } \\
\text { oxycedrus }\end{array}$ & $\begin{array}{l}\text { Genévrier } \\
\text { oxycédre }\end{array}$ & $\begin{array}{l}\text { El arâar - el } \\
\text { gadi }\end{array}$ & $\begin{array}{l}\text { Thérapeutique/ } \\
\text { Cosmétique }\end{array}$ & Infusion & Soin des cheveux, et neurologique \\
\hline 45 & & $\begin{array}{l}\text { Tetraclinis } \\
\text { articulata }\end{array}$ & $\begin{array}{l}\text { Thuya de } \\
\text { Berbérie }\end{array}$ & $\begin{array}{l}\text { El arâar - } \\
\text { Azougaâ }\end{array}$ & Thérapeutique & Infusion & $\begin{array}{l}\text { Traitement des maux de tête, et la } \\
\text { résine qu'elle contient sont utilisées } \\
\text { dans la production de la peinture. }\end{array}$ \\
\hline 46 & \multirow[t]{2}{*}{ Dryopteridaceae } & $\begin{array}{l}\text { Polystichum } \\
\text { aculeatum }\end{array}$ & $\begin{array}{l}\text { Polystic à } \\
\text { aiguillons ou } \\
\text { Aspidie à } \\
\text { soies raides }\end{array}$ & Afersiou & Thérapeutique & Infusion & Affection rénale \\
\hline 47 & & $\begin{array}{l}\text { Polystichum } \\
\text { setiferum }\end{array}$ & & Afersiou & Thérapeutique & Infusion & Affection rénale \\
\hline 48 & Ericaceae & $\begin{array}{l}\text { Arbutus } \\
\text { unedo }\end{array}$ & Arbousier & $\begin{array}{l}\text { Bakhenou -El } \\
\text { matroun - } \\
\text { Sasnou }\end{array}$ & Thérapeutique & Décoction/ Cru & $\begin{array}{l}\text { Utiliser pour la confection de } \\
\text { confitures et de pâtisseries L'écorce } \\
\text { brun rouge est diurétique. En } \\
\text { décoction, sa racine est utilisée contre } \\
\text { l'hypertension. On lui attribue des } \\
\text { propriétés anti-inflammatoires, il est } \\
\text { également efficace contre les } \\
\text { rhumatismes. Les feuilles, l'écorce et } \\
\text { le fruit sont réputés astringents, et } \\
\text { efficaces pour stopper la diarrhée } \\
\text { prise en décoction4. }\end{array}$ \\
\hline $\mathbf{N}^{\circ}$ & Familles & Espèces & $\begin{array}{l}\text { Nom } \\
\text { Français }\end{array}$ & $\begin{array}{l}\text { Nom } \\
\text { Vernaculaire }\end{array}$ & Usage de la plante & $\begin{array}{l}\text { Mode de } \\
\text { préparation }\end{array}$ & Type de maladie \\
\hline
\end{tabular}

${ }^{4}$ a et b Dictionnaire universel des drogues simples [archive], Nicolás Lemery ; https://fr.wikipedia.org/wiki/Arbousier . 
Rhattas et al. J. Appl. Biosci. 2016 Étude ethnobotanique des plantes médicinales dans le Parc National de Talassemtane (Rif occidental du Maroc)

\begin{tabular}{|c|c|c|c|c|c|c|c|}
\hline 49 & Ericaceae & $\begin{array}{l}\text { Erica } \\
\text { terminalis }\end{array}$ & $\begin{array}{l}\text { Bruyère de } \\
\text { Corse }\end{array}$ & El khalnaj & Thérapeutique & Infusion & Affection génito-urinaire \\
\hline 50 & \multirow[t]{2}{*}{ Euphorbiaceae } & $\begin{array}{l}\text { Euphorbia } \\
\text { characias }\end{array}$ & $\begin{array}{l}\text { Euphorbe } \\
\text { characias }\end{array}$ & Anaghou & Thérapeutique & Cataplasme & $\begin{array}{l}\text { Affection cardio-vasculaire, comme } \\
\text { elle présente une toxicité en cas de } \\
\text { contact avec les yeux ou avec la } \\
\text { peau. }\end{array}$ \\
\hline 51 & & $\begin{array}{l}\text { Ricinus } \\
\text { communis }\end{array}$ & Ricin & $\begin{array}{l}\text { El kharwaâ - } \\
\text { Ouryour }\end{array}$ & Cosmétique & Macération & Soin des cheveux \\
\hline 52 & \multirow{8}{*}{ Fabaceae } & $\begin{array}{l}\text { Anthyllis } \\
\text { polycephala }\end{array}$ & & Enafla & Non déterminé & Non déterminé & Non déterminé \\
\hline 53 & & $\begin{array}{l}\text { Anthyllis } \\
\text { vulneraria }\end{array}$ & $\begin{array}{l}\text { Anthyllide } \\
\text { vulnéraire }\end{array}$ & Enafla & Thérapeutique & Décoction & Contre les vertiges \\
\hline 54 & & $\begin{array}{l}\text { Astragalus } \\
\text { armatus }\end{array}$ & Astragale & $\begin{array}{l}\text { Chouk El } \\
\text { guedad }\end{array}$ & Cosmétique & Macération & Massage \\
\hline 55 & & $\begin{array}{l}\text { Cassia } \\
\text { acutifolia }\end{array}$ & $\begin{array}{l}\text { Séné, Séné } \\
\text { d'inde }\end{array}$ & Sena bida & Cosmétique & Macération & $\begin{array}{l}\text { Affection oculaire Soin des cheveux, } \\
\text { et pour perdre du poids }\end{array}$ \\
\hline 56 & & $\begin{array}{l}\text { Ceratonia } \\
\text { siliqua }\end{array}$ & Caroubier & El kharoub & Alimentaire & Décoction & $\begin{array}{l}\text { Utilisée en alimentation, et elle a un } \\
\text { effet régulateur sur la fonction } \\
\text { intestinale et est utilisée dans les cas } \\
\text { de diarrhée. }\end{array}$ \\
\hline 57 & & $\begin{array}{l}\text { Genista } \\
\text { quadriflora }\end{array}$ & $\begin{array}{l}\text { Genêt à } 4 \\
\text { fleurs }\end{array}$ & $\begin{array}{l}\text { Echouk - } \\
\text { Elouki - } \\
\text { Jenista }\end{array}$ & Cosmétique & Décoction & Introduit dans le kohol \\
\hline 58 & & $\begin{array}{l}\text { Ononis } \\
\text { natrix }\end{array}$ & $\begin{array}{l}\text { Bugrane, } \\
\text { Ononis }\end{array}$ & El ounounis & Insecticides & Cataplasme & Insecticide \\
\hline 59 & & $\begin{array}{l}\text { Tripodion } \\
\text { tetraphyllum }\end{array}$ & $\begin{array}{l}\text { Anthyllis à } \\
\text { feuille par } 4\end{array}$ & & Non déterminé & Non déterminé & Non déterminé \\
\hline 60 & \multirow[b]{2}{*}{ Gentianaceae } & $\begin{array}{l}\text { Centaurium } \\
\text { erythraea }\end{array}$ & $\begin{array}{l}\text { Petite } \\
\text { centaurée }\end{array}$ & $\begin{array}{l}\text { Rbiâa elhaya } \\
\text { / Nouara } \\
\text { elhaya }\end{array}$ & Thérapeutique & Décoction & $\begin{array}{l}\text { Affection gastrique et hépatique, } \\
\text { herbe à la fièvre }\end{array}$ \\
\hline 61 & & $\begin{array}{l}\text { Geranium } \\
\text { maculatum }\end{array}$ & & Mrirou (Bâali) & Thérapeutique & Cru & Affection rhumatologique \\
\hline
\end{tabular}


Rhattas et al. J. Appl. Biosci. 2016 Étude ethnobotanique des plantes médicinales dans le Parc National de Talassemtane (Rif occidental du Maroc)

\begin{tabular}{|c|c|c|c|c|c|c|c|}
\hline $\mathbf{N}^{\circ}$ & Familles & Espèces & $\begin{array}{l}\text { Nom } \\
\text { Français }\end{array}$ & $\begin{array}{l}\text { Nom } \\
\text { Vernaculaire }\end{array}$ & Usage de la plante & $\begin{array}{l}\text { Mode de } \\
\text { préparation }\end{array}$ & Type de maladie \\
\hline 62 & Iridaceae & $\begin{array}{l}\text { Crocus } \\
\text { sativus }\end{array}$ & $\begin{array}{l}\text { Safran } \\
\text { cultivé }\end{array}$ & Safran Beldi & $\begin{array}{l}\text { Thérapeutique/ } \\
\text { Cosmétique }\end{array}$ & Cru & $\begin{array}{l}\text { Soins de visage et affection } \\
\text { métabolique }\end{array}$ \\
\hline 63 & & Ajuga iva & $\begin{array}{l}\text { Germandrée } \\
\text { musquée }\end{array}$ & $\begin{array}{l}\text { Chandkoura/ } \\
\text { Tadjich } \\
=\text { Toute Bari }\end{array}$ & $\begin{array}{l}\text { Cosmétique/ } \\
\text { Thérapeutique/ alimentaire }\end{array}$ & Macération/ Cru & $\begin{array}{l}\text { Affection oculaire Soin des cheveux } \\
\text { Maladie hépatique, maladie du froid }\end{array}$ \\
\hline 64 & & $\begin{array}{l}\text { Calamintha } \\
\text { officinalis }\end{array}$ & Calament & $\begin{array}{l}\text { Halhal } \\
=\text { Khouzama }\end{array}$ & Thérapeutique & Infusion/ Cuit & $\begin{array}{l}\text { Affection génito-urinaire et soin des } \\
\text { cheveux/ Affection digestive et } \\
\text { Affection respiratoire }\end{array}$ \\
\hline 65 & & $\begin{array}{l}\text { Lavandula } \\
\text { dentata }\end{array}$ & $\begin{array}{l}\text { Lavande à } \\
\text { feuille } \\
\text { dentée }\end{array}$ & $\begin{array}{l}\text { Halhal } \\
=\text { Khouzama }\end{array}$ & $\begin{array}{l}\text { Thérapeutique/ } \\
\text { Cosmétique }\end{array}$ & $\begin{array}{l}\text { Infusion/ } \\
\text { Macération }\end{array}$ & $\begin{array}{l}\text { Affection rhumatologique/Affection } \\
\text { génito-urinaire/ Affection oculaire Soin } \\
\text { des cheveux }\end{array}$ \\
\hline 66 & & $\begin{array}{l}\text { Lavandula } \\
\text { stoechas }\end{array}$ & Lavande & $\begin{array}{l}\text { Halhal } \\
=\text { Khouzama }\end{array}$ & $\begin{array}{l}\text { Thérapeutique/ } \\
\text { Cosmétique }\end{array}$ & Macération & $\begin{array}{l}\text { Soin des cheveux et Affection génito- } \\
\text { urinaire }\end{array}$ \\
\hline 67 & & $\begin{array}{l}\text { Marrubium } \\
\text { fontianum }\end{array}$ & Marrube & El mariwa & Cosmétique & Infusion & $\begin{array}{l}\text { Affection cutanée, irritation pour la } \\
\text { peau et les muqueuses gastriques }\end{array}$ \\
\hline 68 & Lamiaceae & $\begin{array}{l}\text { Marrubium } \\
\text { vulgare }\end{array}$ & $\begin{array}{l}\text { Marrube } \\
\text { blanc ou } \\
\text { Marrube } \\
\text { Commun }\end{array}$ & $\begin{array}{l}\text { Mchichtro - } \\
\text { Tafergana }\end{array}$ & Thérapeutique & Infusion & $\begin{array}{l}\text { Affection digestive/ Affection } \\
\text { rhumatologique }\end{array}$ \\
\hline 69 & & $\begin{array}{l}\text { Melissa } \\
\text { officinalis }\end{array}$ & Melissa & Tourenjan & Thérapeutique & Cuit/ Infusion & $\begin{array}{l}\text { Affection digestive et remède pour les } \\
\text { intestins }\end{array}$ \\
\hline 70 & & $\begin{array}{l}\text { Mentha } \\
\text { pulegium }\end{array}$ & $\begin{array}{l}\text { Menthe } \\
\text { pouliot }\end{array}$ & $\begin{array}{l}\text { Flyou - } \\
\text { Naânaâ - } \\
\text { Mantha }\end{array}$ & Thérapeutique & $\begin{array}{l}\text { Infusion/ } \\
\text { Macération }\end{array}$ & $\begin{array}{l}\text { Affection respiratoire/ Affection } \\
\text { digestive }\end{array}$ \\
\hline 71 & & $\begin{array}{l}\text { Origanum } \\
\text { grosii }\end{array}$ & Origan & Zâatar & Thérapeutique & Infusion & Affection digestive \\
\hline 72 & & $\begin{array}{l}\text { Origanum } \\
\text { majorana }\end{array}$ & $\begin{array}{l}\text { Marjolaine } \\
\text { origan / la } \\
\text { menthe } \\
\text { citronnée }\end{array}$ & $\begin{array}{l}\text { Merdadouch } \\
=\text { Merdekouch }\end{array}$ & Thérapeutique/Cosmétique & Infusion & $\begin{array}{l}\text { Affection neurologique et maladie de } \\
\text { la bouche/ Affection digestive }\end{array}$ \\
\hline 73 & & $\begin{array}{l}\text { Rosmarinus } \\
\text { officinalis }\end{array}$ & Romarin & Azir & Thérapeutique & Infusion/ Cuit & $\begin{array}{l}\text { Affection neurologique, de foie et } \\
\text { hémostatique/guérie Alzheimer }\end{array}$ \\
\hline
\end{tabular}


Rhattas et al. J. Appl. Biosci. 2016 Étude ethnobotanique des plantes médicinales dans le Parc National de Talassemtane (Rif occidental du Maroc)

\begin{tabular}{|c|c|c|c|c|c|c|c|}
\hline $\mathbf{N}^{\circ}$ & Familles & Espèces & $\begin{array}{l}\text { Nom } \\
\text { Français }\end{array}$ & $\begin{array}{l}\text { Nom } \\
\text { Vernaculaire }\end{array}$ & $\begin{array}{l}\text { Usage de la } \\
\text { plante }\end{array}$ & $\begin{array}{l}\text { Mode de } \\
\text { préparation }\end{array}$ & Type de maladie \\
\hline 74 & \multirow{5}{*}{ Lamiaceae } & $\begin{array}{c}\text { Salvia } \\
\text { argentea }\end{array}$ & $\begin{array}{c}\text { Sauge } \\
\text { argentée }\end{array}$ & El kouissa & Thérapeutique & Infusion & Affection génito-urinaire \\
\hline 75 & & $\begin{array}{l}\text { Salvia } \\
\text { officinalis }\end{array}$ & La sauge & Salmia & Thérapeutique & Infusion & $\begin{array}{l}\text { Les troubles hormonaux, et douleurs des règles/ } \\
\text { Affection génito-urinaire }\end{array}$ \\
\hline 76 & & Satureja alpina & Sarriette & $\begin{array}{l}\text { Zâaytra - } \\
\text { Tazouknit }\end{array}$ & Thérapeutique & Infusion & Affection digestive \\
\hline 77 & & $\begin{array}{l}\text { Thymus } \\
\text { algeriensis }\end{array}$ & Thym & $\begin{array}{l}\text { Zîtra - } \\
\text { tazouknit - } \\
\text { Mantha }\end{array}$ & Thérapeutique & Cru & Affection digestive \\
\hline 78 & & Thymus zygis & Thym salsa & Zïtra & Thérapeutique & Infusion & Affection Intestinale \\
\hline 79 & Lauraceae & Laurus nobilis & Laurier-sauce & $\begin{array}{l}\text { Warakate } \\
\text { sidna mousa } \\
\text { - El ghar }\end{array}$ & Thérapeutique & Cuit & $\begin{array}{l}\text { Affection digestive, Ses feuilles sont utilisées en } \\
\text { cuisine pour leur arôme. }\end{array}$ \\
\hline 80 & Liliaceae & $\begin{array}{l}\text { Asparagus } \\
\text { officinalis }\end{array}$ & Asperge & Sekoum & Thérapeutique & Décoction & Affection rénale, Affection du foie, rhumatisme \\
\hline 81 & Lythraceae & $\begin{array}{l}\text { Lythrum } \\
\text { hyssopifolia }\end{array}$ & Lythrum & $\begin{array}{l}\text { Rayhan } \\
\text { elmaa - } \\
\text { sabon elmaa }\end{array}$ & Thérapeutique & Infusion & Affection neurologique \\
\hline 82 & Myrsinaceae & $\begin{array}{l}\text { Anagallis } \\
\text { arvensis }\end{array}$ & $\begin{array}{l}\text { Le mouron } \\
\text { rouge }\end{array}$ & Anagallis & $\begin{array}{l}\text { Cosmétique/ } \\
\text { Thérapeutique }\end{array}$ & $\begin{array}{l}\text { Décoction/ } \\
\text { cataplasme }\end{array}$ & $\begin{array}{l}\text { Soin des cheveux, traitement de certaines } \\
\text { maladies respiratoires. }\end{array}$ \\
\hline 83 & Myrtaceae & $\begin{array}{l}\text { Myrtus } \\
\text { communis }\end{array}$ & $\begin{array}{l}\text { Myrte } \\
\text { commun }\end{array}$ & $\begin{array}{l}\text { Rayhan - Ass } \\
\text { - Elhbak }\end{array}$ & $\begin{array}{l}\text { Thérapeutique/ } \\
\text { Cosmétique }\end{array}$ & $\begin{array}{l}\text { Infusion/ } \\
\text { Macération/ } \\
\text { Cuit }\end{array}$ & $\begin{array}{l}\text { Affection oculaire Soin des cheveux/ Affection } \\
\text { digestive, et neurologique }\end{array}$ \\
\hline 84 & \multirow[b]{2}{*}{ Oleaceae } & $\begin{array}{l}\text { Jasminum } \\
\text { officinale }\end{array}$ & Jasmin blanc & Jawhare dar & Thérapeutique & Infusion & Affection neurologique \\
\hline 85 & & Olea europaea & $\begin{array}{l}\text { L'olivier } \\
\text { sauvage - } \\
\text { Oléastre }\end{array}$ & $\begin{array}{l}\text { Zaytoune } \\
\text { Bari }\end{array}$ & Thérapeutique & Macération & $\begin{array}{l}\text { Affection pulmonaire et maladie de diabète/ } \\
\text { Affection digestive }\end{array}$ \\
\hline $\mathbf{N}^{\circ}$ & Familles & Espèces & $\begin{array}{l}\text { Nom } \\
\text { Français }\end{array}$ & $\begin{array}{l}\text { Nom } \\
\text { Vernaculaire }\end{array}$ & $\begin{array}{l}\text { Usage de la } \\
\text { plante }\end{array}$ & $\begin{array}{l}\text { Mode de } \\
\text { préparation }\end{array}$ & Type de maladie \\
\hline 86 & Paeoniaceae & $\begin{array}{l}\text { Paeonia } \\
\text { coriacea }\end{array}$ & $\begin{array}{l}\text { Pivoine } \\
\text { sauvage }\end{array}$ & $\begin{array}{l}\text { El fawanya - } \\
\text { Ouard zouan }\end{array}$ & $\begin{array}{l}\text { Thérapeutique/ } \\
\text { Cosmétique }\end{array}$ & $\begin{array}{l}\text { Décoction/ } \\
\text { Infusion }\end{array}$ & $\begin{array}{l}\text { Régularise les règles et les hormones chez la } \\
\text { femme, Affection Cutanée, Affection Digestive }\end{array}$ \\
\hline 87 & Papaveraceae & Fumaria & Fumeterre & DEBABA & Non déterminé & Non & Non déterminé \\
\hline
\end{tabular}


Rhattas et al. J. Appl. Biosci. 2016 Étude ethnobotanique des plantes médicinales dans le Parc National de Talassemtane (Rif occidental du
Maroc)

\begin{tabular}{|c|c|c|c|c|c|c|c|}
\hline & & macrosepala & & & & déterminé & \\
\hline 88 & & $\begin{array}{l}\text { Papaver } \\
\text { rhoeas }\end{array}$ & $\begin{array}{l}\text { Grand } \\
\text { Coquelicot }\end{array}$ & $\begin{array}{l}\text { El } \\
\text { khachkhach - } \\
\text { Belnaâman }\end{array}$ & Thérapeutique & Macération & $\begin{array}{l}\text { Narcotique, pectoral, adoucissant, } \\
\text { antispasmodique, hypnotique, magie }\end{array}$ \\
\hline 89 & & $\begin{array}{l}\text { Papaver } \\
\text { setigerum }\end{array}$ & $\begin{array}{l}\text { Poppy de } \\
\text { Troie ou Nain } \\
\text { Bread seed } \\
\text { Poppy }\end{array}$ & $\begin{array}{l}\text { El } \\
\text { khachkhach }\end{array}$ & Thérapeutique & Macération & $\begin{array}{l}\text { Effets analgésiques, largement utilisées dans } \\
\text { l'alimentation }\end{array}$ \\
\hline 90 & \multirow[t]{2}{*}{ Pinaceae } & $\begin{array}{l}\text { Abies } \\
\text { maroccana }\end{array}$ & $\begin{array}{l}\text { Sapin du } \\
\text { Maroc }\end{array}$ & $\begin{array}{l}\text { Chouh } \\
\text { Marocain - } \\
\text { Sanawbar }\end{array}$ & Thérapeutique & Infusion & Bronchite aigüe, Abcès et gangrène pulmonaire \\
\hline 91 & & Pinus nigra & $\begin{array}{l}\text { Pin noir de } \\
\text { Maurétanie }\end{array}$ & $\begin{array}{l}\text { Tayda - } \\
\text { Tighrou }\end{array}$ & Cosmétique & Infusion & Soin des cheveux \\
\hline 92 & Poaceae & $\begin{array}{l}\text { Hordeum } \\
\text { murinum }\end{array}$ & Orge des rats & Khartal & Pâturage & Cru & Alimentation des bétails \\
\hline 93 & \multirow{2}{*}{ Polygonaceae } & Rumex crispus & Oseille & $\begin{array}{l}\text { El hamad - } \\
\text { Hmida - } \\
\text { Tasemoumt }\end{array}$ & Alimentaire & Cru/ cuit & Affection digestive, Antidiabétique \\
\hline 94 & & Rumex pulcher & Oseille & $\begin{array}{l}\text { El hamad - } \\
\text { Homida - } \\
\text { Tasemoumt }\end{array}$ & Alimentaire & Cru/ cuit & $\begin{array}{l}\text { Frotter une feuille de Rumex sur une peau } \\
\text { piquée par les orties ou les insectes supprime les } \\
\text { démangeaisons. }\end{array}$ \\
\hline 95 & Saxifragaceae & $\begin{array}{l}\text { Saxifraga tri } \\
\text { crenata }\end{array}$ & Saxifrage & Kaser Lahjer & Alimentaire & Crut & Alimentation \\
\hline 96 & $\begin{array}{l}\text { Scrophulariaceae } \\
\text { / Plantaginaceae }\end{array}$ & $\begin{array}{l}\text { Antirrhinum } \\
\text { majus }\end{array}$ & $\begin{array}{l}\text { Gueule-de- } \\
\text { loup, muflier }\end{array}$ & El khatmya & Thérapeutique & Cataplasme & Anti-inflammatoire \\
\hline $\mathrm{N}^{\circ}$ & Familles & Espèces & $\begin{array}{l}\text { Nom } \\
\text { Français }\end{array}$ & $\begin{array}{l}\text { Nom } \\
\text { Vernaculaire }\end{array}$ & $\begin{array}{l}\text { Usage de la } \\
\text { plante }\end{array}$ & $\begin{array}{l}\text { Mode de } \\
\text { préparation }\end{array}$ & Type de maladie \\
\hline 97 & \multirow{3}{*}{ Solanaceae } & Atropa baetica & $\begin{array}{l}\text { Belladone } \\
\text { bétique }\end{array}$ & Bleydour & Thérapeutique & Cru & $\begin{array}{l}\text { A faible dose peut être utilisé contre les } \\
\text { affections neurologiques }\end{array}$ \\
\hline 98 & & $\begin{array}{l}\text { Atropa } \\
\text { belladonna }\end{array}$ & Belladone & Bleydour & Thérapeutique & Cru & Antiasthmatique \\
\hline 99 & & $\begin{array}{l}\text { Hyoscyamus } \\
\text { albus }\end{array}$ & $\begin{array}{l}\text { Jusquiame } \\
\text { blanche }\end{array}$ & $\begin{array}{l}\text { kingat - El } \\
\text { banj Abyad }\end{array}$ & Thérapeutique & Cru & $\begin{array}{l}\text { Affection digestive/ Affection cutané et soin de } \\
\text { brûlure }\end{array}$ \\
\hline 100 & Taxaceae & Taxus baccata & If & Daghes & Thérapeutique & Infusion & Traitement de rhumatisme \\
\hline 101 & Thymelaeaceae & Daphne & Bois gentil & Matnan - & Cosmétique & Cataplasme/ & Affection oculaire Soin des cheveux \\
\hline
\end{tabular}


Rhattas et al. J. Appl. Biosci. 2016 Étude ethnobotanique des plantes médicinales dans le Parc National de Talassemtane (Rif occidental du Maroc)

\begin{tabular}{|l|l|l|l|l|l|l|l|}
\hline & gnidium & & $\begin{array}{l}\text { Lezaz - } \\
\text { Dafna }\end{array}$ & Macération & \\
\hline 102 & Urticaceae & $\begin{array}{l}\text { Urtica } \\
\text { membranacea }\end{array}$ & Ortie & $\begin{array}{l}\text { El hourika el } \\
\text { malsaa }\end{array}$ & $\begin{array}{l}\text { Thérapeutique/ } \\
\text { Cosmétique }\end{array}$ & $\begin{array}{l}\text { Infusion/ } \\
\text { Macération/ } \\
\text { Cataplasme }\end{array}$ & soin des cheveux et guérison de l'anémie \\
\hline 103 & Verbenaceae & $\begin{array}{l}\text { Verbena } \\
\text { officinalis }\end{array}$ & Verveine & Elouiza & Thérapeutique & Infusion & Affection neurologique \\
\hline
\end{tabular}


Familles d'usage fréquent: Concernant les espèces médicinales les plus utilisées par la population enquêtée (Fig. $n^{\circ} 6$ ), 103 espèces appartenant à 47 familles sont utilisées à des fins thérapeutiques. Les familles les plus représentées et les plus utilisées par cette population sont respectivement les Lamiaceae $(42,35 \%)$, les Cistaceae $(30,60 \%)$, suivie par les Asteraceae (9,25\%), ensuite par les Apocynaceae, et les Thymelaeaceae qui représentent respectivement $8,90 \%$. Fennane \&. Ibn Tattou (2012), inventaire de la flore vasculaire du Maroc, ont conclu dans leur catalogue que les trois premières familles de la flore vasculaire marocaine sont toujours les mêmes et dans le même ordre Asteraceae, Fabaceae et Poaceae et totalisent 1329 espèces, soit plus du tiers de l'inventaire spécifique national ; alors que notre résultat a montré que les Asteraceae représentent $9,25 \%$ dans le Parc. Les mêmes auteurs ont signalé que trois autres familles (Brassicaceae, Lamiaceae, Caryophyllaceae) sont considérées comme plus riches en espèces, 200 espèces, dont les Lamiaceae représentent $40 \%$ et occupent le 6ème rang sur le plan richesse spécifique. Dans le Parc national de Talassemtane, les représentants de cette famille totalisent également $42,35 \%$.

\section{Répartition de la richesse spécifique des familles botaniques}

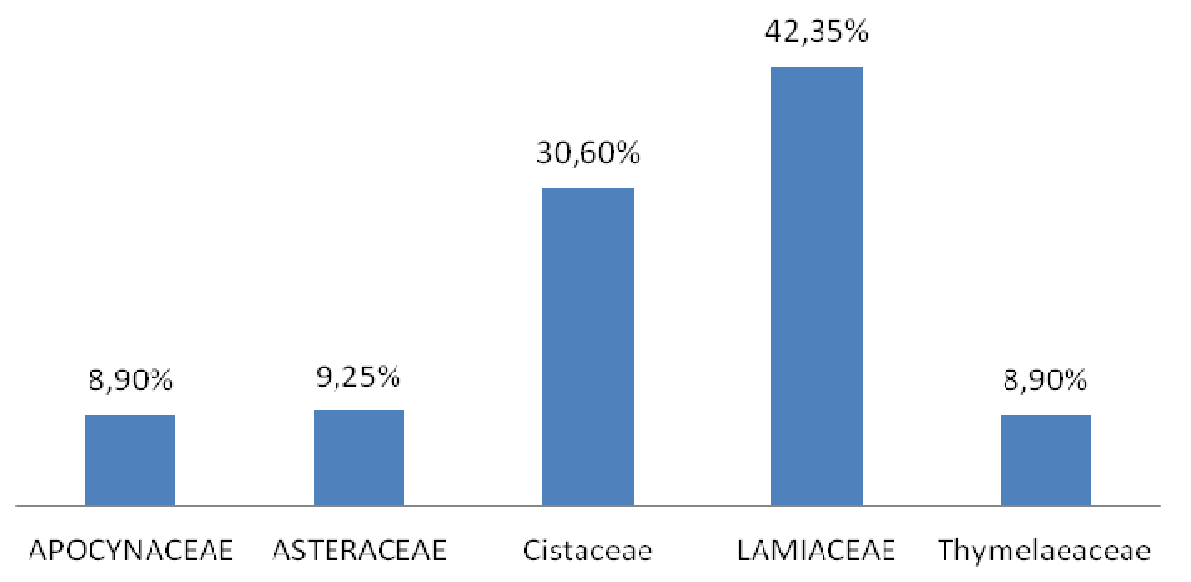

Figure 6 : Familles botaniques les plus représentées dans la zone étudiée (Enquête début mois de mars au fin août 2014) ;

Espèces d'usages fréquents: L'analyse de l'information collectée (Figure $n^{\circ} 7$ ) montre que les espèces Cistus varius Pourret et Marrubium vulgare sont représentées par $14,59 \%$ chacune, ce qui signifie que 280 informateurs ont signalé leur utilisation, suivies de Mentha pulegium, avec $12,46 \%$, et Matricaria chamomilla avec 9,25\%, Cistus monspeliencis, Daphne gnidium, et Nerium oleander représentent respectivement $8,90 \%$ chacune, ensuite viennent Salvia officinalis avec $7,83 \%$, suivie par Lavandula dentata avec $7,47 \%$, et enfin Cistus albidus avec $7,12 \%$. 


\section{Fréquence des espèces les plus utilisées}

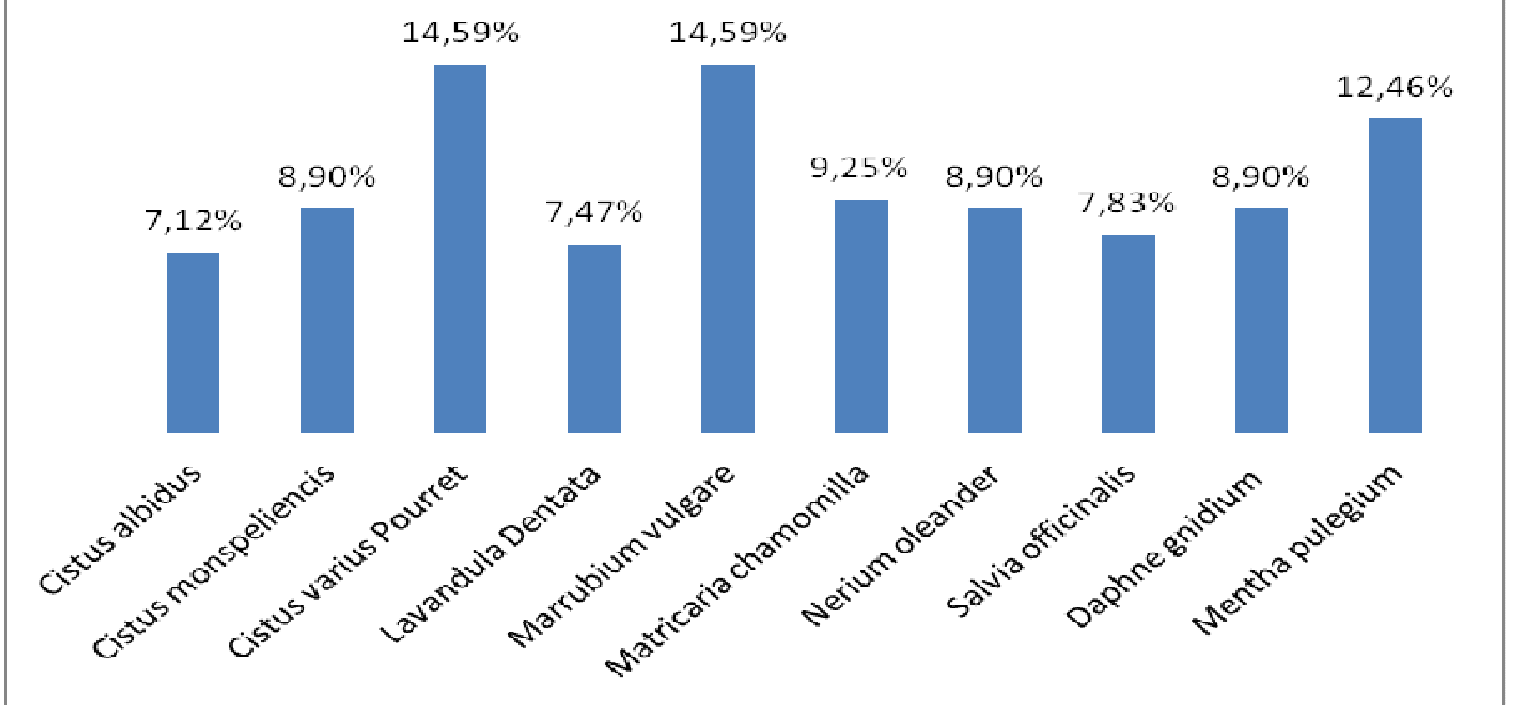

Figure 7 : Fréquence des espèces les plus utilisées (Enquête début mois de Mars au fin août 2014) ;

Indications thérapeutiques: L'analyse ethnobotanique de la Figure $n^{\circ} 8$ nous a permis de déterminer également les maladies traitées par les plantes médicinales et utilisées d'une manière traditionnelle dans la région étudiée. Ce sont les affections digestives qui sont les plus traitées avec les plantes médicinales, avec un pourcentage de $37,78 \%$, suivies par les affections cutanées (15\%), ensuite viennent les autres affections génito-urinaires $(8,89 \%)$, les affections neurologiques (6,67\%), les affections rénales, les soins des cheveux et les plantes utilisées en alimentation sont représentés respectivement par $4,44 \%$ chacune. Cistus varius Pourret est utilisée contre les lésions cutanées, Marrubium vulgare et Mentha pulegium L. sont utilisés contre les affections de l'appareil digestif, Lavandula dentata contre les affections génito-urinaires et Matricaria camomilla contre les affections neurologiques, ce qui explique la pression particulière exercée sur ces espèces végétales.

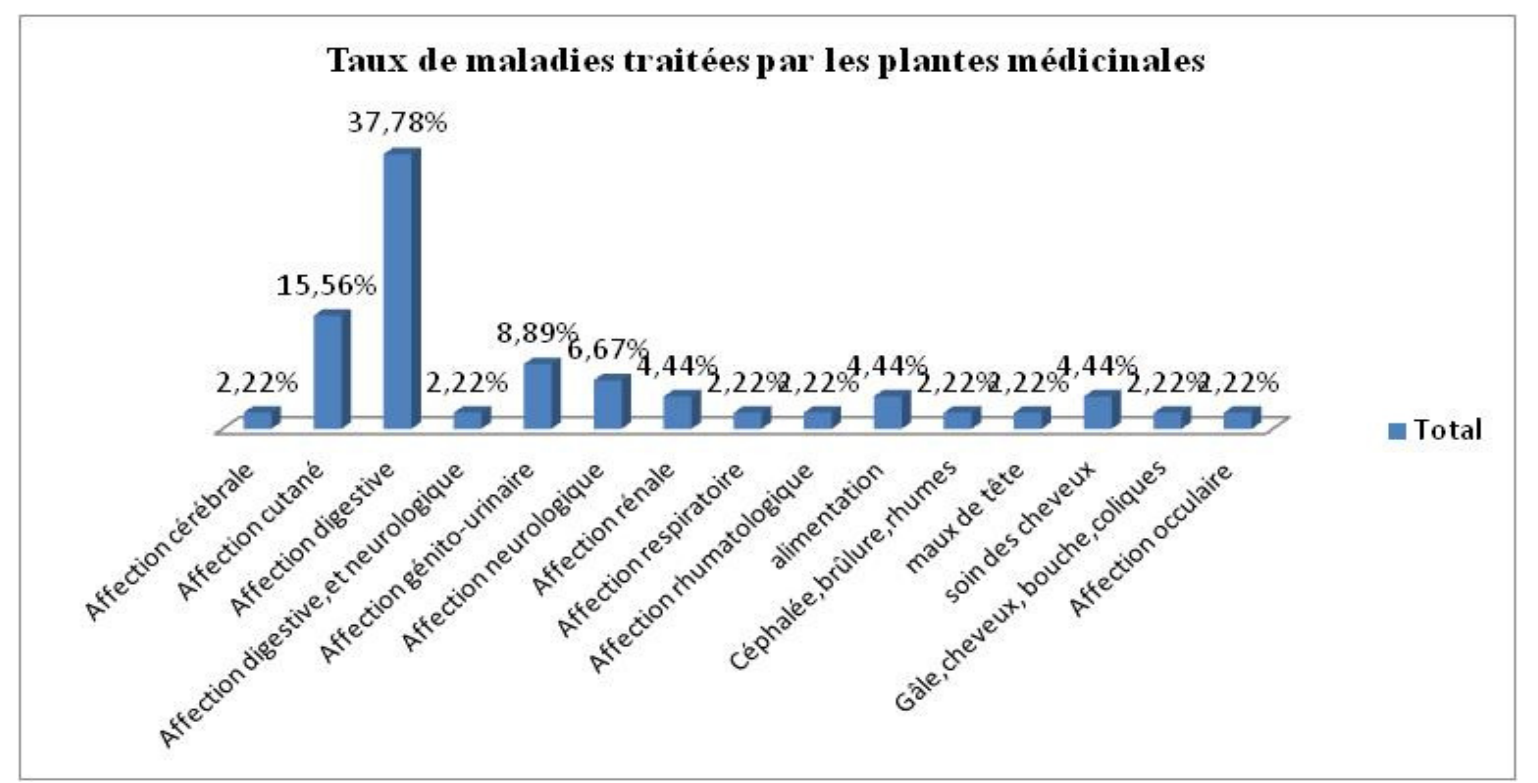

Figure 8 : Taux de maladies traitées par les plantes médicinales (Enquête début mois de mars au fin août 2014) ; 
Parties utilisées : Selon les résultats de la figure $n^{\circ} 9$, la partie la plus utilisée de la plante c'est la feuille, avec un pourcentage de $71,75 \%$. La fréquence d'utilisation élevée de feuilles peut être expliquée par l'aisance et la rapidité de la récolte (Bitsindou, 1986), mais aussi par le fait qu'elles sont le siège de la photosynthèse et parfois du stockage des métabolites secondaires responsables des propriétés biologiques de la plante (Bigendako-Polygenis \& Lejoly, 1990). L'utilisation des feuilles ne représente aucun danger pour la régénération des plantes et assurera la conservation de la richesse floristique; en effet, il existe une relation manifeste entre la partie utilisée de la plante exploitée et les effets de cette exploitation sur son existence (Cunningham 1996). Le mode de cueillette compromet sérieusement la durabilité des espèces médicinales surtout les bulbeuses (Mehdioui \& Kahouadji, 2007). Le Parc national de Talassemtane est riche et diversifié en plantes médicinales et il suffit de les exploiter d'une manière rationnelle.

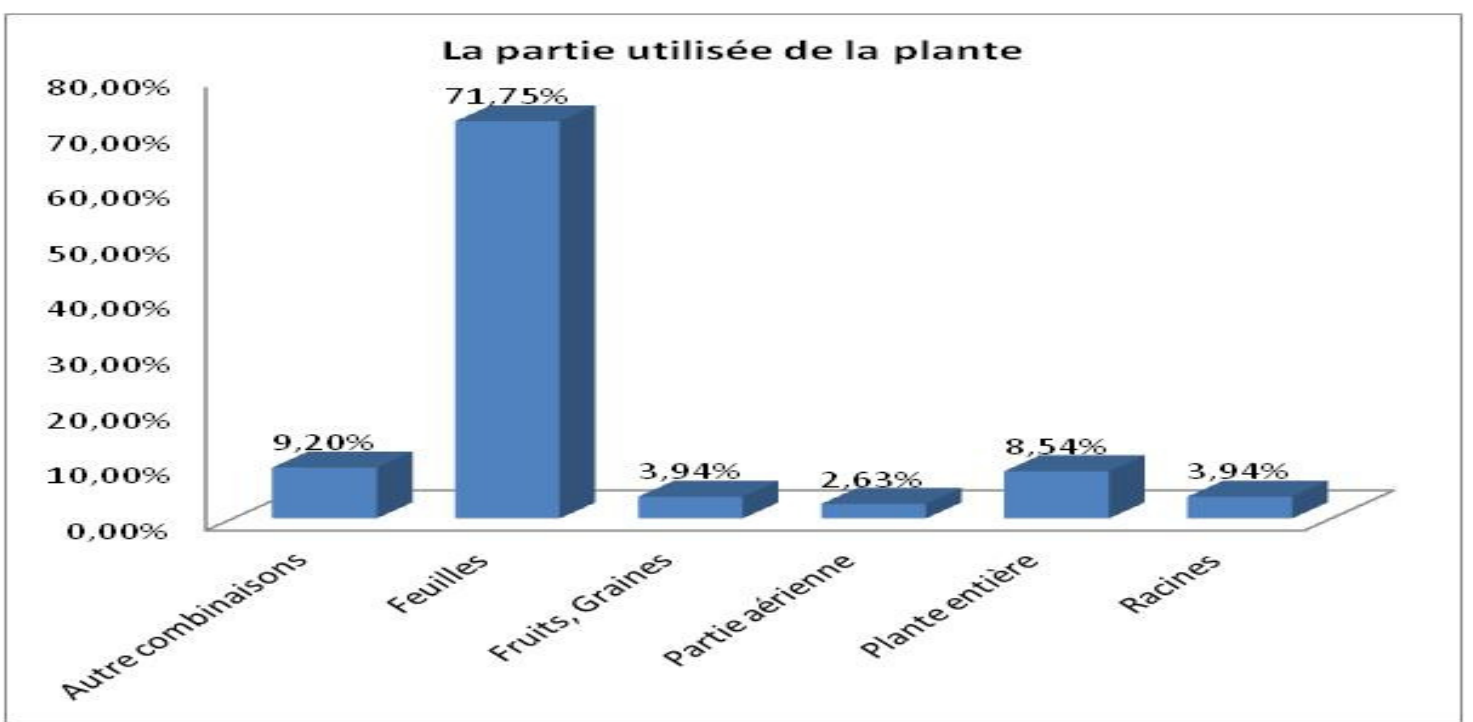

Figure 9 : La partie utilisée de la plante (Enquête début mois de mars au fin août 2014);

Mode de préparation: Les modes d'utilisation, les plus répandus (Figure $n^{\circ} 10$ ), sont classés comme suit: l'infusion, macération, cru, cataplasme et décoction, avec respectivement $30,43 \% ; 14,41 \%$; $10,75 \% ; 10,32 \%$; et $7,85 \%$. La meilleure utilisation d'une plante serait celle qui en préserverait toutes les propriétés tout en permettant l'extraction et l'assimilation des principes actives (Dextreit, 1984). De plus, les plantes médicinales ont des effets indésirables quand elles sont pratiquées de façon incorrecte par les patients. De ce fait, la médecine douce doit être pratiquée avec précaution et à l'intérieur des paramètres et des mesures bien précises (Benlamdini et al., 2014). 
Mode de préparation des plantes médicinales

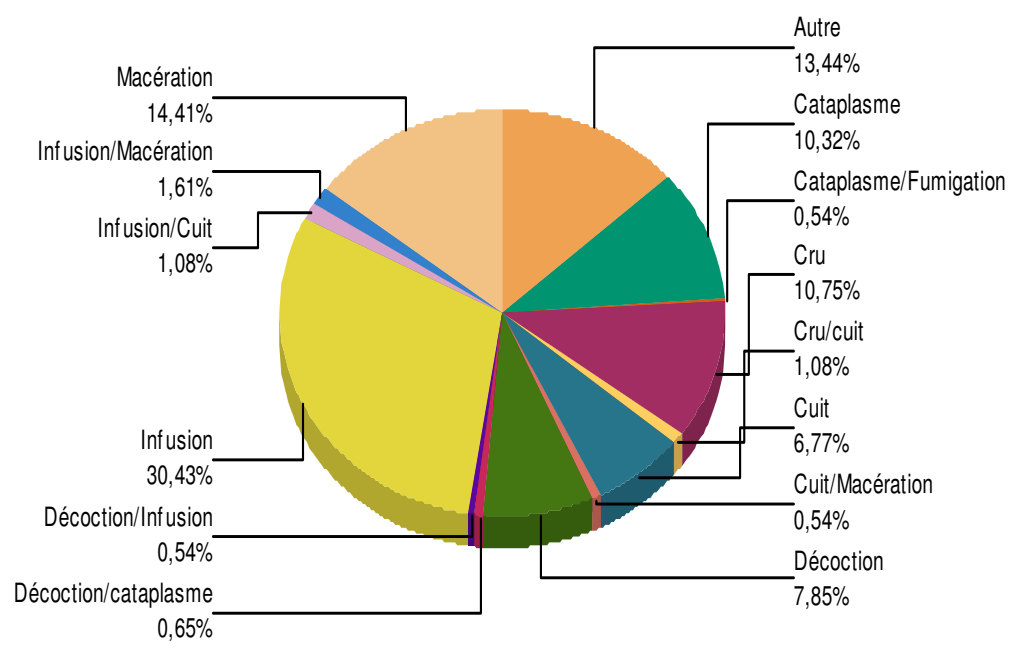

\begin{tabular}{|lr|}
\hline & $0,00 \%$ \\
Autre & $13,44 \%$ \\
Cataplasme & $10,32 \%$ \\
Cataplasme/Fumigation & $0,54 \%$ \\
Cru & $10,75 \%$ \\
Cru/cuit & $1,08 \%$ \\
Cuit & $6,77 \%$ \\
Cuit/Macération & $0,54 \%$ \\
Décoction & $7,85 \%$ \\
Décoction/cataplasme & $0,65 \%$ \\
Décoction/Inf usion & $0,54 \%$ \\
Infusion & $30,43 \%$ \\
Infusion/Cuit & $1,08 \%$ \\
Infusion/Macération & $1,61 \%$ \\
Macération & $14,41 \%$ \\
Total : & $100,00 \%$ \\
\hline
\end{tabular}

Figure 10 : Mode de préparation des plantes médicinales (Enquête début mois de mars au fin août 2014);

\section{CONCLUSION}

La fréquence d'utilisation des plantes médicinales par la population locale du Parc est fonction du profil des personnes enquêtées. Ainsi, la fréquence chez les jeunes âgés de 20 à 39 ans est de $47 \%$, alors qu'elle est de l'ordre de $17 \%$ pour les personnes âgées de 60 à 79 ans. Les femmes et les hommes ont un savoir médicinal partagé avec un léger avantage pour les femmes $(57 \%)$. Par ailleurs, l'analyse floristique des résultats obtenus a permis de recenser 103 espèces et 82 genres appartenant à 47 familles. Parmi ces dernières, les Lamiaceae $(42,35 \%)$, les Cistaceae $(30,60 \%)$ et les Asteraceae $(9,25 \%)$, les Apocynaceae et Thymelaeaceae qui représentent respectivement $(8,90 \%)$ chacune, dominent la flore médicinale dans le

\section{RÉFÉRENCES BIBLIOGRAPHIQUES}

Bammi J., 1995- Activités antibactérienne et antivirale de quelques huiles essentielles extraites de plantes médicinales. Diplôme d'Études Approfondies. Univ. Tunis II., Fac. Sc. Tunis, $63 p$

Bammi J. et Douira A., 2002. Les plantes médicinales dans la forêt de l'Achach (Plateau Central, Maroc). Acta Botánica Malacitana, 27 : 131 145

Bammi. J. Gmira N., Douira A., Remmal A., Khelifa R., et Manai M., -2000- A study of antibacterial and antiviral activities of essential oils of parc de Talassemtane. Cette étude montre également que les maladies les plus traitées par ces plantes médicinales sont dominées par les affections digestives $(37,78 \%)$, les affections cutanées $(15,56 \%)$, les affections génito-urinaires $(8,89 \%)$ et les affections neurologiques $(6,67 \%)$. Pour leur part, les recettes thérapeutiques sont préparées essentiellement par infusion $(30,43 \%)$ et macération $(14,41 \%)$. En définitive, les résultats de cette étude pourraient constituer une base de données pour les recherches ultérieures dans le domaine de la phytochimie dans le but d'identifier de nouveaux principes actifs naturels utilisables en pharmacologie.

oregano and clove. The first African Congress on Biology and Health. 23, 24, 25 April, 2000. Ferhat Abbas, University. Setif, Algeria.

Bammou M., Daoudi A., Slimani I., Najem M., El Houssine Bouiamrine E. H., Ibijbijen J. et Nassiri L., 2014.Valorisation du lentisque «Pistacia lentiscus L. »: Étude ethnobotanique, Screening phytochimique et pouvoir antibactérien. Journal of Applied Biosciences, 86 : 7966 - 7975

Belkamel A., Bammi J., Janneot V., Belkamel A., Dehbi Y., et Douira A., 2008. Evaluation de la 
biomasse et analyse des huiles essentielles de trois variétés de basilic (Ocimum basilicum L.) cultivées au Maroc. Acta Bot. Gallica, 155 (4), 467-476.

Belkamel A., Bammi J., Belkamel A., et Douira A., 2013. Étude de la composition chimique de l'huile essentielle d'une endémique Ibéromarocaine: Origanum compactum (Benth.). Journal of Animal \& Plant Sciences, 19 (1) : 2880-2887

Benabid A., 1983. Études biogéographique et dynamique des peuplements forestiers du Rif (Maroc). Annales de la Recherche Forestière au Maroc, 1 (23) : 49-129.

Benabid A., 1987. Les grands écosystèmes terrestres et leur préservation. G.E.M., Rabat, $4: 177$ 190.

Benabid A., et Bellakhdar J., 1987. Relevés floristiques et catalogue des plantes médicinales dans le Rif occidental (missions ethnobotaniques 1984-1987). Al Biruniya. Rev. Mar. Pharm., 3 (2) : 87-120.

Benabid A., 1991. La préservation de la forêt au Maroc. In M. Rejdali \& V.H. Heywood Eds., Conservation des ressources végétales, Actes Editions, Rabat, p : 97-104.

Bellakhdar J., Baayoui A. Kazdari A. et Marechal J., 1987. Herboristes et médecine traditionnelle à Tissint, oasis présaharien du sud marocain (province de Tata). Al Biruniya. Rev. Mar. Pharm., 3 (1) : 7-50.

Benchaâbane A., \& Abbad A., 1997. Les plantes médicinales commercialisées à Marrakech. Ed. Info, Marrakech, 74 pp.

Benlamdini N., Elhafian M., Rochdi A., et Zidane L., 2014. Étude floristique et ethnobotanique de la flore médicinale du Haute Moulouya, Maroc. Journal of Applied Biosciences, 78: 6771 6787

Bigendako-Polygenis M.J. \& Lejoly, J., 1990. La pharmacopée traditionnelle au Burundi. Pesticides et médicaments en santé animale. Pres. Univ. Namur., pp. 425-442.

Bitsindou M., 1986. Enquête sur la phytothérapie traditionnelle à Kindamba et Odzala (Congo) et analyse de convergence d'usage des plantes médicinales en Afrique centrale. Mem. Doc (inéd.). Univ. Libre de Bruxelles, 482 pp.

Cunningham A.B., 1996. Peuples, parc et plantes. Recommandations pour les zones à usages multiples et les alternatives de développement autour du parc Naturel de Bwindi Impénétrable, Ouganda. Documents de travail Peuples et Plantes $n^{\circ}$ 4. UNESCO, Paris, 66 p.

Dextreit R., 1984. La cure végétale, Toutes les plantes pour se guérir, Vivre en harmonie, $3{ }^{\text {èmeed, }} 118$ p.

El Hassani M., Douiri E. M., Bammi J., Zidane L., Badoc A., Douira A., 2013. Plantes médicinales de la Moyenne Moulouya (NordEst du Maroc). Ethno pharmacologia, $n^{\circ} 50$

Fennane M., \& Ibn Tatou M., 2012: Statistiques et commentaires sur l'inventaire actuel de la flore vasculaire du Maroc. Bulletin de l'Institut Scientifique, Rabat, section Sciences de la Vie, 34 (1) :. 1-9.

Hmamouchi, M., 2001.Les plantes médicinales et aromatiques marocaines, 2ème éd., 389 pp.

Hseini S., \& Kahouadji A., 2007. Étude ethnobotanique de la flore médicinale dans la région de Rabat (Maroc occidental). Lazaroa, 28 : 79-93.

Kahouadji M.S., 1995. Contribution à une étude ethnobotanique des plantes médicinales dans

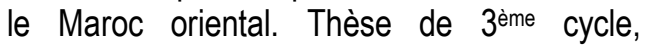
Université Mohamed 1er, Faculté des Sciences, Oujda, Maroc, $205 \mathrm{p}$.

Lahsissene H., Kahouadji A., Tijane M., et Hseini S., 2009. Catalogue des plantes médicinales utilisées dans la région de Zaër (Maroc occidental). Lejeunia, 186, 1- 2.

Lespinasse P., 1975. Géologie des zones externes et des flyschs entre Chaouène et Zoumi (centre de la chaîne rifaine, Maroc). Thèse de Doct. État, Université de Toulouse I, $248 \mathrm{p}$.

Mehdioui R., \& Kahouadji A., 2007. Étude ethnobotanique auprès de la population riveraine de la forêt d'Amsittène : cas de la Commune d'Imi n'Tlit (Province d'Essaouira). Bulletin de l'Institut Scientifique, Rabat, section Sciences de la Vie, 29 : 11-20.

Michard A., 1976. Éléments de géologie Marocaine. Serv. Géol. Rabat, n³00, $233 \mathrm{p}$.

Ouchbani T., 2012. Étude de la phytochimie et la pharmacognosie de substances naturelles et de métabolites secondaires bioactives issus des champignons endophytiques et des plantes médicinales d'origine marocaine: Caractérisation chimique et biopharmaceutique Thèse de Doctorat, Université Mohamed V, Faculté des Sciences, Rabat, Maroc, 150p. 
PDAGPNT, 2004. Plan directeur d'aménagement et de gestion du Parc national de Talassemtane, équipe du projet étude et plans de gestion des Aires Protégées du Maroc - BCEOM/SECA en collaboration avec Administration des Eaux et Forêts et de la conservation des sols et ensemble de tous les services forestiers provinciaux, $p: 13$

Quezel P., Barbero M., \& Loisel R., 1990. Les reboisements en région méditerranéenne. Incidences biologiques et économiques. Forêt méditerranéenne, 1.12 (2) : 103-114.

Reille M., 1977. Contribution pollen analytique à I'histoire holocène de la végétation des montagnes du Rif (Maroc Septentrional). Rech. franç. Sur le Quaternaire, INQUA 1977, Suppl. Bull. A.F.E.Q., 1977-1 (50) : 53-76.

Remmal, A., 1994. Activités antibactérienne et antivirale des huiles essentielles d'origan, de girofle et de thym. Thèse de Doctorat d'état es sciences naturelles. Unv. Sidi Mohammed Ben Abdallah. Fès, Maroc.
Sauvage $\mathrm{CH} ., 1958$. Intérêt biogéographique du BouHachem (Rif occidental). Société des Sciences Naturelles et Physiques du Maroc, t. 38 (I er trimestre) : 17-26.

Settaf A., El Kabbaj S., Labhal A., Cherrah Y., Slaoui A. \& Hassar M., 2000. Herniaria hirsuta reduce biliary cholesterol in dogs. Induced changes in bile composition. Biologique et Santé, 1 (I) :.44-49.

Taiqui L. \& Martin C.C., 1997. Éléments historiques d'analyse écologique des paysages montagneux du Rif occidental (Maroc). Mediterránea. Serie d'estudios biológicos, 16: 23-35.

Tantaoui - Elaraki A., Lattaoui N., \& Errifi A., 1993 aComposition and antimicrobial activity of the essential oils of Thymus broussonetti, T. zygis and T. satureioides. J. Essent. Oil. Res., 5: 4553.

Tantaoui - Elaraki A., Ferhout H. \& Errifi A., 1993 b. Inhibition of the fungal asexual reproduction stages by three Moroccan essential oils. J. Essent. Oil. Res., 5 : 535-545.

Annexe : 1

Questionnaire Plantes médicinales et phytothérapie :

Date :

Commune

Daouar :

Auteur :

dit :

Informations concernant l'informateur :

Genre : Masculin $\square \quad$ Féminin $\square$

Age :.....

Niveau d'étude : Analphabète $\square \quad$ Primaire $\square$ Secondaire $\square$ Universitaire $\square$

Profession :

Situation familiale : Célibataire $\square$ Mariéa

Lorsque vous vous sentez malade, vous vous adressez :

- $\quad$ À la médecine traditionnelle $\square$

Pourquoi : Efficace $\square \quad$ Moins cher $\square \quad$ Médicaments inefficaces $\square$

- $\quad$ À la médecine moderne $\square$

Pourquoi : Efficace $\square \quad$ Plus précise $\square$ Toxicité des plantes $\square$

- $\quad$ Si c'est les deux, quelle est la première : Médecin moderne $\square$ Médecin traditionnelle $\square$

Matériel végétal :

Nom vernaculaire :

Nom scientifique :

Type de plante : Sauvage $\square \quad$ Cultivée $\square \quad$ Adventice $\square$

Usage de la plante : Thérapeutique

Cosmétiquea Autres : 


\section{Rhattas et al. J. Appl. Biosci. $2016 \quad$ Étude ethnobotanique des plantes médicinales dans le Parc National de Talassemtane (Rif occidental du Maroc)}

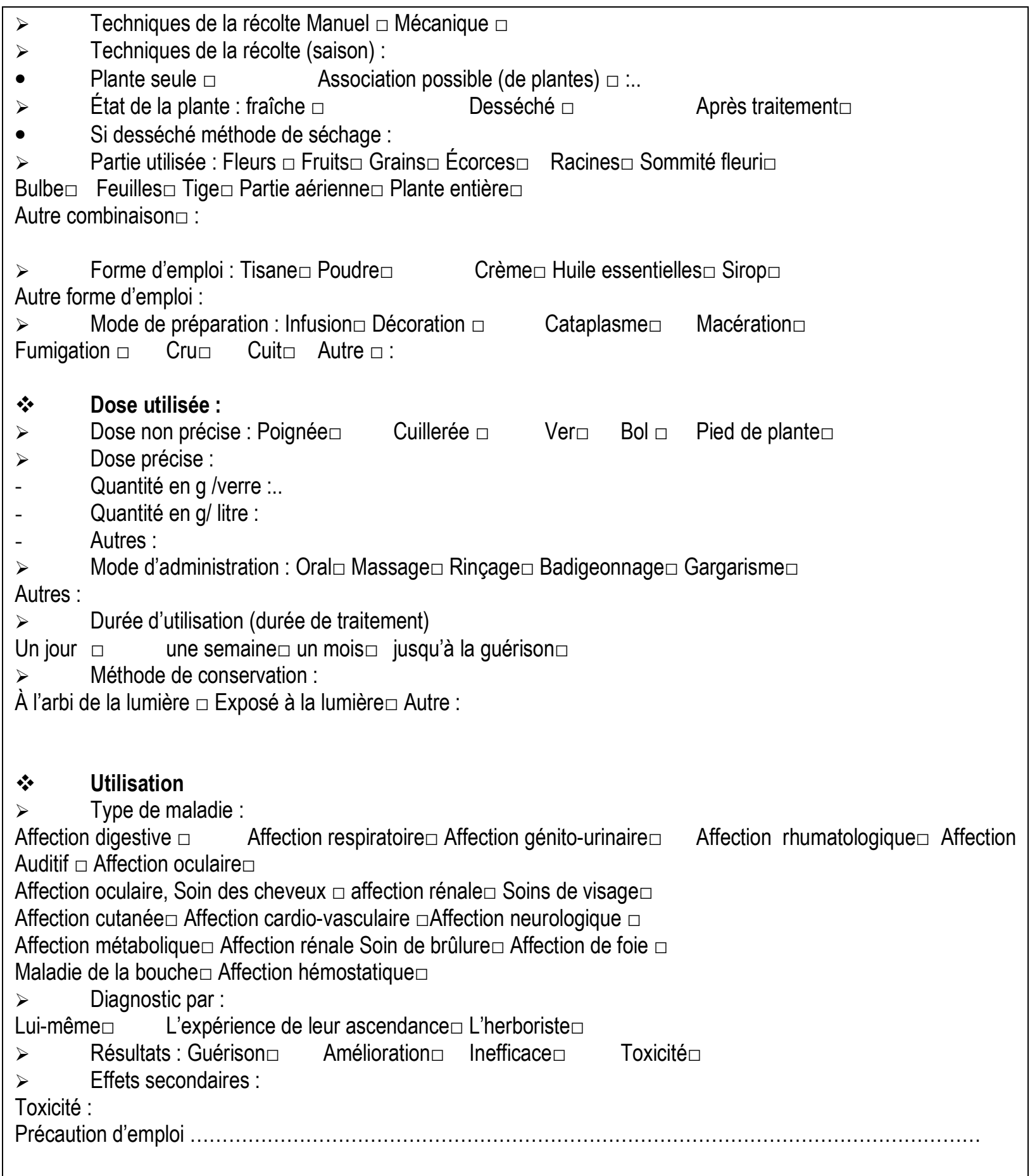

